\title{
Low-Field Mobility and Galvanomagnetic Properties of Holes in Germanium with Phonon Scattering
}

\author{
Lawætz, Peter
}

Published in:

PHYSICAL REVIEW

Link to article, DOI:

10.1103/PhysRev.174.867

Publication date:

1968

Document Version

Publisher's PDF, also known as Version of record

Link back to DTU Orbit

Citation (APA):

Lawætz, P. (1968). Low-Field Mobility and Galvanomagnetic Properties of Holes in Germanium with Phonon Scattering. PHYSICAL REVIEW, 174(3), 867-880. https://doi.org/10.1103/PhysRev.174.867

\section{General rights}

Copyright and moral rights for the publications made accessible in the public portal are retained by the authors and/or other copyright owners and it is a condition of accessing publications that users recognise and abide by the legal requirements associated with these rights.

- Users may download and print one copy of any publication from the public portal for the purpose of private study or research.

- You may not further distribute the material or use it for any profit-making activity or commercial gain

- You may freely distribute the URL identifying the publication in the public portal

If you believe that this document breaches copyright please contact us providing details, and we will remove access to the work immediately and investigate your claim. 
the data at low temperature would not be valid if they yield results which are inconsistent with the result obtained from the analysis of the $\pi_{l}(\rho)$-versus- $T$ data [Fig. 6 and Eq. (4)]. Evidently, the simple model based on the pressure dependence of a single donor level does not suffice to explain the piezoresistance data around $20^{\circ} \mathrm{K}$. A detailed experimental investigation in this temperature range is required before any firm explanation can be proposed for this disagreement between the low- and the high-temperature piezoresistance data.

\section{CONCLUSION}

The result $\pi_{\text {shear }} \approx 0$ indicates that the lowest conduction-band minimum for this material is situated at $\mathbf{k}=(0,0,0)$, and is in agreement with other work. ${ }^{1,3-6}$ The piezo-Hall and piezoresistance effects can be qualitatively explained on the basis of the simple model that the donor ionization energy $\epsilon_{d}$ increases appreciably with pressure. The value $\epsilon_{d} \approx 0.019 \mathrm{eV}$ determined for one sample from the data on $\pi_{l}(\rho)$ versus $T$ is in excellent agreement with the value $\epsilon_{d} \approx 0.020 \mathrm{eV}$ estimated from the low-temperature data on $\rho$ versus $T$. The result $\left(d \epsilon_{d} / d P\right) \geq+5.2 \times 10^{-12} \mathrm{eV} / \mathrm{dyn}^{-2}$ derived from the pressure data at $195^{\circ} \mathrm{K}$ suggests that there is considerable contribution from some band other than the (000) conduction band to the donor state functions. The piezoresistance data around $20^{\circ} \mathrm{K}$, when simply interpreted on the basis of the pressure dependence of the ionization energy of the single donor level, yield $\left(d \epsilon_{d} / d P\right) \approx+2.6 \times 10^{-12} \mathrm{eV} / \mathrm{dyn}^{-2}$, in disagreement with the estimate obtained from the pressure data at $195^{\circ} \mathrm{K}$.

\title{
Low-Field Mobility and Galvanomagnetic Properties of Holes in Germanium with Phonon Scattering
}

\author{
P. LAWAETZ \\ Physics Laboratory III, The Technical University of Denmark, Lyngby, Denmark
}

(Received 3 June 1968)

\begin{abstract}
A theoretical calculation of the low-field galvanomagnetic properties of holes in Ge has been carried out incorporating all relevant details of the band structure. The scattering is limited to acoustic and optical phonons and is described by the deformation potentials $a, b, d$, and $d_{0}$. For pure acoustic scattering, no overall consistency is found between available galvanomagnetic data and deformation potentials derived directly from experiments on strained Ge. The discrepancies may be ascribed to ionized-impurity scattering, but at higher temperatures where optical phonon scattering is operative, the deviations are still appreciable. We are led to conclude that the deformation-potential theory of phonon scattering needs reconsideration, and a nontrivial correction is pointed out.
\end{abstract}

\section{INTRODUCTION}

$\mathrm{A}^{\mathrm{T}}$ LTHOUGH the electrical transport properties of holes in Ge are more or less qualitatively understood, their quantitative interpretation is still far from satisfactory. ${ }^{1}$ The difficulties encountered in this connection are primarily due to the complicated energy spectrum of holes in the vicinity of the degenerate valence-band edge, which consists of the two bands

$$
\begin{aligned}
& E_{1,2}(\mathbf{k}) \\
& \quad=A k^{2} \pm\left[B^{2} k^{4}+C^{2}\left(k_{x}{ }^{2} k_{y}{ }^{2}+k_{y}{ }^{2} k_{z}{ }^{2}+k_{z}{ }^{2} k_{x}{ }^{2}\right)\right]^{1 / 2} .
\end{aligned}
$$

The principal aim of the present work is to calculate the low-field galvanomagnetic parameters mobility, Hall factor, and magnetoresistance, avoiding any serious approximations in the band structure or its effects on scattering, so that it might be possible to establish a

1 E. G. S. Paige, Progress in Semiconductors (Heywood and Co., Ltd., London, 1964), Vol. 8, p. 1. This review article discusses most of the earlier experimental and theoretical work on electrical transport properties of holes in Ge. quantitative correlation between some important transport properties.

The scattering mechanisms will be limited to acoustic and optical phonons, and are treated on the basis of the deformation-potential theory developed by $\mathrm{Bir}$ and Pikus. ${ }^{2}$ In this theory, the hole-phonon interaction is determined by the four deformation potentials $a, b, d$, and $d_{0}$. Of these, the first three, pertaining to the acoustic scattering, also describe the change in the valence-band structure with static strain ${ }^{3}$ and, consequently, they enter the theory of a large number of phenomena pertaining to the valence band. $d_{0}$ is connected with the optical phonon scattering and appears only in the theory of transport phenomena.

The previous theories of low-field transport may all be regarded as simplifications of the general case intro-

${ }^{2}$ G. L. Bir and G. E. Pikus, Fiz. Tverd. Tela 2, 2287 (1960) [English transl.: Soviet Phys.-Solid State 2, 2039 (1961)]. A similar theory was worked out independently by $\mathbf{M}$. Tiersten, IBM J. Res. Develop. 5, 122 (1961).

${ }^{3}$ G. E. Pikus and G. L. Bir, Fiz. Tverd. Tela 1, 1624 (1959) [English transl.: Soviet Phys.-Solid State 1, 1502 (1960)]. 
duced by some special choice of the parameters $A, B, C$, $a, b, d$, and $d_{0}{ }^{4}$ These models fall in the following five groups (only pure acoustic scattering is considered):

(a) Single spherical band, $B=C=0 ;$ (1) isotropic scattering, ${ }^{1} b=d=0$.

(b) Two spherical bands, $C=0$; (1) isotropic scattering, ${ }^{1} b=d=0$, (2) anisotropic scattering, ${ }^{5} d=b \sqrt{3}$.

(c) Real band structure, Eq. (1.1); (1) isotropic scattering, ${ }^{6} b=d=0,(2)$ anisotropic scattering, general case.

Only the mobility calculation by Tiersten ${ }^{7}$ belongs to the general case (c2). The inadequacy of the simplified models may be illustrated by a comparison with the experimental value of the acoustic Hall factor $r=\mu_{H} / \mu_{0}$ $=1.36 .^{8-10}$ The corresponding theoretical results are (a1) $1.18,{ }^{1}$ (b1) $2.51,{ }^{11}$ (b2) $1.60 \leq r \leq 2.53,{ }^{5}$ (c1) $2.07 .{ }^{6}$ With the exception of the simplest and least-realistic case (a1), all models studied so far yield a Hall factor above the experimental value. A similar situation exists for the transverse magnetoresistance, and no model based on spherical bands predicts a nonvanishing longitudinal magnetoresistance. Consequently, there is a clear need for a theory incorporating the full complexity of the band structure and the acoustic phonon scattering.

It is well established experimentally ${ }^{1,12}$ that the temperature dependence of the drift mobility $\mu_{0}$ of holes in $\mathrm{Ge}$ is of the form $T^{-2.3}$ in the range $120-300^{\circ} \mathrm{K}$. Since this deviates considerably from the $T^{-1.5}$ dependence to be expected from pure acoustic scattering and parabolic bands, ${ }^{7,13}$ either strong optical phonon scattering ${ }^{1,12}$ or nonparabolicity ${ }^{1,14}$ has been suggested as a possible cause, but so far no theory has incorporated both mechanisms simultaneously. This will be done in the present work, and for the first time the temperature dependence of the galvanomagnetic effects will be calculated and compared with experimental data.

The physical model on which the present calculations are based is outlined in Sec. II. Particular emphasis will be laid on a qualitative discussion of the hole tran-

\footnotetext{
${ }^{4}$ In the course of the present calculations for Ge, it has been shown that the usual approximation of an isotropic acoustic phonon spectrum leads to a negligible error, especially in the models where anisotropy is already present in band structure $(C \neq 0)$ or scattering $(b, d \neq 0)$.

${ }^{5}$ G. L. Bir, E. Normantas, and G. E. Pikus, Fiz. Tverd. Tela 4, 1180 (1962) [English transl.: Soviet Phys.-Solid State 4, 867 (1962)].

${ }^{6}$ B. Lax and J. G. Mavroides, Phys. Rev. 100, 1650 (1955); J. G. Mavroides and B. Lax, ibid. 107, 1530 (1957); 108, 1648 (E) (1957).

${ }^{7}$ M. Tiersten, J. Phys. Chem. Solids 25, 1151 (1964).

${ }^{8}$ C. Goldberg, E. N. Adams, and R. E. Davis, Phys. Rev. 105,865 (1957).

${ }^{9}$ V. I. Stafeev and V. M. Tuchkevich, Zh. Tekh. Fiz. 28 , 1642 (1958) [English transl.: Soviet Phys.-Tech. Phys. 3, 1513 (1958)]

${ }_{10}$ A. C. Beer and R. K. Willardson, Phys. Rev. 110, 1286 (1958).

${ }^{11}$ E. M. Conwell, Phys. Rev. 94, 1416 (1954)

${ }_{12}$ D. M. Brown and R. Bray, Phys. Rev. 127, 1593 (1962).

${ }^{13}$ P. Lawaetz, Phys. Status Solidi 11, K117 (1965).

${ }_{14}$ R. Keiper and H. W. Streitwolf, Phys. Status Solidi 12, K21 (1965).
}

sition rates due to acoustic phonon scattering and of the validity of some necessary approximations in the treatment of these quantities.

The central problem in transport theory is the determination of the distribution function from the Boltzmann equation. As a consequence of the complexity of the basic model, the solution will be performed numerically. In Sec. III, we show how the Boltzmann equation may be brought into a form convenient for numerical treatment by a power-series expansion of the distribution function in the external fields, retaining first-order terms in electric field and second-order terms in magnetic field. The angular dependence of all quantities entering the transport equation is handled by series expansions in a suitable set of harmonics. In Sec. III B, the link between the distribution function and the macroscopic transport parameters is established. Section III C deals with the problem of including the nonparabolicity of the light hole band in the frame of the theory.

The main problem in the numerical computation is the evaluation of the various coefficients belonging to the series expansions in surface harmonics of the angular-dependent quantities. This problem is closely connected with the truncation of the series in question, and thus with the over-all accuracy of the calculation. The details are discussed in Sec. IV, in order to establish that the principal uncertainty of the present results is eventually due to the basic physical model and not to approximations in the computational procedure. In an earlier paper, ${ }^{15}$ we have stressed the important simplifications introduced by direct application of the inherent cubic symmetry of the problem, and these ideas are further developed in Sec. IV.

In Sec. V, we present the results of calculations of mobility, the Hall factor, and of transverse and longitudinal magnetoresistance. In the case of pure acoustic scattering, a fairly complete mapping of all possible combinations of the deformation potentials $a, b$, and $d$ is shown. This is made possible by the simple temperature dependence of the transport parameters in question, following from the usual assumptions of parabolic bands and the equipartition of acoustic phonon energies. It is shown that most of the results can be interpreted in terms of a simple model with unequal relaxation times in the two bands. If nonparabolicity and optical phonon scattering are included, a complete representation of the results would be hopeless were it not for the fact that the temperature dependence of the transport parameters is practically independent of the details of the acoustic scattering and is a function only of the lowtemperature value (pure acoustic scattering). A further simplification is introduced by an automatic adjustment of the optical deformation potential $d_{0}$ so that the value of the mobility at $300^{\circ} \mathrm{K}$ coincides with the experimental result.

\footnotetext{
${ }^{15}$ P. Lawaetz, Phys. Rev. 166, 763 (1968), referred to as I.
} 
The crucial point of the theory is reached in Sec. IV, where we compare the results of the present theory with existing experimental data. Here, a careful selection of data must be made in order to ensure that they actually represent pure phonon scattering and low fields. It will then be found that, although the fit is considerably improved as compared to more simplified models, there are significant discrepancies. Furthermore, we may compare the results with deformation potentials obtained by static strain experiments and this leads to an enhanced inconsistency of the galvanomagnetic results.

Finally, Sec. VII contains a discussion of possible explanations for this rather surprising insufficiency of the present model. It is concluded that deformationpotential theory does not give the complete picture of lattice scattering, at least of the part due to optical phonons

\section{THEORETICAL MODEL}

\section{A. Hole Energy Spectrum}

The energy spectrum $E=E(\mathbf{k})$ of holes in $\mathrm{Ge}$ is derived by $\mathbf{k} \cdot \mathbf{p}$ perturbation theory including spin and spin-orbit coupling. ${ }^{16}$ In the vicinity of the valenceband edge at $\mathbf{k}=0$, the spectrum consists of two bands degenerate at $\mathbf{k}=0$ and a third band split off $\Delta=0.295$ $\mathrm{eV}^{17}$ by the spin-orbit coupling. Since the spin-orbit splitting $\Delta$ is large compared to thermal energies at $300^{\circ} \mathrm{K}$, the split-off band may be neglected in transport theory, but the proximity of this band induces a considerable $k^{4}$ term in the nearest band, i.e., in the light hole band.

To second order in $\mathbf{k}$, the energy spectrum is given by expression (1.1). This is the parabolic approximation, where

$$
\begin{aligned}
E & =k^{2} g_{\lambda}(\hat{k}), \\
g_{\lambda}(\hat{k}) & =A+(-1)^{\lambda}\left[B^{2}+C^{2}\left(\hat{k}_{x}{ }^{2} \hat{k}_{y}{ }^{2}+\hat{k}_{y}{ }^{2} \hat{k}_{z}{ }^{2}+\hat{k}_{z}{ }^{2} \hat{k}_{x}{ }^{2}\right)\right]^{1 / 2} .
\end{aligned}
$$

$\hat{k}$ is the unit vector in the direction of $\mathbf{k} ; \lambda=1$ and 2 give the heavy and the light hole band, respectively. $A, B$, and $C$ have been measured by cyclotron resonance, and we shall use the values ${ }^{18}$

$$
\begin{aligned}
A & =13.27 \pm 0.025, \\
B & =8.63 \pm 0.12, \\
|C| & =12.4 \pm 0.25,
\end{aligned}
$$

in units of $\hbar^{2} / 2 m$, where $m$ is the free electron mass. Values similar to (2.3) have been obtained by others. ${ }^{19}$

${ }^{16}$ G. Dresselhaus, A. F. Kip, and C. Kittel, Phys. Rev. 98, 368 (1955); E. O. Kane, J. Phys. Chem. Solids 1, 82 (1956).

${ }_{17}$ A. C. Baynham and E. G. S. Paige, in Proceedings of the Seventh International Conference on the Physics of Semiconductors, Paris, 1964 (Academic Press Inc., New York, 1964), p. 149.

${ }_{18}$ B. W. Levinger and D. R. Frankl, J. Phys. Chem. Solids 20, 281 (1961).

19 R. R. Goodman, Phys. Rev. 122, 397 (1961); J. C. Hensel, Solid State Commun. 4, 231 (1966).
The sign of $A$ pertains to positive hole energies. The sign of $B$ and the related quantity $D$ defined below will be discussed later. For the present, we shall take them to be positive. Insertion of (2.3) in (2.2) shows that the light hole band is nearly spherically symmetric.

When the split-off band is taken into account directly, the second-order $\mathbf{k} \cdot \mathbf{p}$ perturbation matrix ${ }^{16}$ cannot be diagonalized in a simple way except for $\mathbf{k}$ parallel to the $\langle 100\rangle$ and $\langle 111\rangle$ directions. For the $\langle 100\rangle$ directions, we obtain $^{20}$

$$
\begin{aligned}
& E_{1}=(A-B) k^{2} \\
& \begin{aligned}
E_{2}= & \frac{1}{2}\left[(2 A+B) k^{2}+\Delta\right]-\left\{\frac{1}{4}\left[(2 A+B) k^{2}+\Delta\right]^{2}\right. \\
& \left.\quad-\Delta(A+B) k^{2}-(A+2 B)(A-B) k^{4}\right\}^{1 / 2}
\end{aligned}
\end{aligned}
$$

For the $\langle 111\rangle$ directions, $B$ is replaced by $D / \sqrt{3},{ }^{20}$ where

$$
D=\left(3 B^{2}+C^{2}\right)^{1 / 2} \text {. }
$$

From (2.4) we observe that the heavy hole band remains parabolic in the principal symmetry directions, whereas (2.5) shows that the light hole band is more affected. An expansion of (2.5) gives

$$
\begin{aligned}
E_{2} & \approx(A+B) k^{2}-2 B^{2} k^{4} / \Delta \\
& =k^{2} g_{2}\left[1-C_{2} k^{2} g_{2} / \Delta\right] .
\end{aligned}
$$

With the same degree of accuracy we may write (2.7) in the form

$$
E_{2}\left(1+C_{2} E_{2} / \Delta\right)=k^{2} g_{2} \text {. }
$$

We now make the approximation of generalizing (2.8) to arbitrary directions of $\mathbf{k}$. For $C_{2}$ we then use a suitable mean value between $C_{2}(100)$ and $C_{2}(111)$ :

$$
C_{2}=B^{2}(A+B)^{-2}+D^{2}(A \sqrt{3}+B)^{-2} .
$$

We have thus chosen a specific angular dependence of the nonparabolic contribution to the light hole band, but in view of the smallness of this correction such a simplifying approximation is justified.

We shall later show how the form (2.8) is taken into account in our calculations. When not specified, the energy spectrum is considered in the parabolic form (2.1).

\section{B. Phonon Spectrum}

Since thermal holes are situated in a band extremum at $\mathbf{k}=0$, it follows that the phonons of interest have very small wave vectors $\mathbf{q}$. The long-wavelength part of the phonon spectrum in Ge consists of three acoustic branches with frequencies $\omega \propto q$ and three optical branches degenerate at $\mathbf{q}=0$ with $\omega$ independent of $q$.

The frequencies $\omega_{\mathrm{q} s}$ and the corresponding polarization vectors $\hat{e}_{s}$ for the acoustic phonons of branch $s$ are the solutions of the secular equation ${ }^{21}$

$$
\sum_{\alpha^{\prime}=1}^{3}\left\{S_{\alpha \alpha^{\prime}}-\rho \omega_{\mathrm{q} s}{ }^{2} \delta_{\alpha \alpha^{\prime}}\right\} \hat{e}_{s \alpha^{\prime}}=0,
$$

${ }^{20} \mathrm{~W}$. Bernard, H. Roth, and W. D. Straub, Phys. Rev. 132, 33 (1963).

${ }_{21}$ W. P. Mason, Physical Acoustics and the Properties of Solids (D. van Nostrand Co., Inc., New York, 1958), p. 370; H. M. J. Smith, Phil. Trans. Roy. Soc. (London) A241, 105 (1948). 
where $\rho$ is the mass density of the crystal and $\mathbf{S}$ is a Shockley matrix ${ }^{21,22}$ with the elements

$$
\begin{aligned}
& S_{i i}=\left(C_{11}-C_{44}\right) q_{i}{ }^{2}+C_{44} q^{2}, \\
& S_{i j}=\left(C_{12}+C_{44}\right) q_{i} q_{j}, \quad i \neq j .
\end{aligned}
$$

$C_{11}, C_{12}$, and $C_{44}$ are the elastic constants for which we shall use the values ${ }^{23}$

$$
\begin{aligned}
& C_{11}=1.30 \times 10^{12} \text { dyn } \mathrm{cm}^{-2}, \\
& C_{12}=0.49 \times 10^{12} \mathrm{dyn}^{-2}, \\
& C_{44}=0.68 \times 10^{12} \text { dyn } \mathrm{cm}^{-2} .
\end{aligned}
$$

The temperature variation of the elastic constants is within $2 \%$ from 77 to $300^{\circ} \mathrm{K} .{ }^{23}$ This variation will not be taken into account.

For a given $\hat{q}$, the numerical evaluation of eigenvalues and eigenvectors of $\mathrm{Eq}$. (2.10) is standard and will not be further commented upon.

The optical phonons are characterized by the feature that to zeroth order in $q$ the two atoms in the unit cell oscillate in opposite phase. The polarization-independent frequency at $q=0$ has been measured by neutron spectrometry ${ }^{24}$ :

$$
\hbar \omega_{0}=0.037 \mathrm{eV} \text {. }
$$

As a consequence of the degeneracy in zeroth order of $q$, the polarization vectors $\hat{e}_{0 s}$ (for one of the atoms) may be any set of three orthogonal unit vectors.

\section{Acoustic Phonon Scattering}

To describe the scattering of holes by acoustic phonons, Bir and Pikus ${ }^{2}$ have worked out a deformationpotential theory, taking into account the complicated energy spectrum of holes. In this theory, the rate of transition from state $\mathbf{k}$ in band $\lambda$ to $\mathbf{k}^{\prime}$ an $\lambda^{\prime}$ is ${ }^{2}$

$$
\begin{aligned}
P_{\mathrm{ac}}\left(\lambda^{\prime} \mathbf{k}^{\prime}, \lambda \mathbf{k}\right)=\sum_{ \pm} \sum_{\mathrm{q} s} & \frac{\pi k_{B} T}{\hbar V \rho \omega_{\mathrm{q} s}{ }^{2}} U\left(\lambda \hat{k}, \lambda^{\prime} \hat{k}^{\prime}, \mathbf{q} s\right) \delta_{\mathrm{k}, \mathbf{k}^{\prime} \pm \mathbf{q}} \\
& \times \delta\left(E_{\lambda^{\prime}}\left(\mathbf{k}^{\prime}\right)-E_{\lambda}(\mathbf{k}) \pm \hbar \omega_{\mathrm{q} s}\right),
\end{aligned}
$$

where $V$ is the volume of the crystal, $k_{B}$ is Boltzmann's constant, and

$$
\begin{aligned}
& U\left(\lambda \hat{k}, \lambda^{\prime} \hat{k}^{\prime}, \mathbf{q} s\right)=\delta E_{\lambda}\left(\hat{k}, \epsilon^{\prime}\right) \delta E_{\lambda^{\prime}}\left(\hat{k}^{\prime}, \epsilon^{\prime}\right)-\delta E_{1}\left(0, \epsilon^{\prime}\right) \\
& \times \delta E_{2}\left(0, \epsilon^{\prime}\right) \Psi\left(\lambda \hat{k}, \lambda^{\prime} \hat{k}^{\prime}\right), \\
& \delta E_{\lambda}\left(\hat{k}, \epsilon^{\prime}\right)=a \epsilon_{x x}{ }^{\prime}+\left[B b \epsilon_{x x}{ }^{\prime}\left(3 \hat{k}_{x}^{2}-1\right)\right. \\
& \left.+2 D d \epsilon_{x y}{ }^{\prime} \hat{k}_{x} \hat{k}_{y}\right] / 2 E_{\hat{k} \lambda}+\text { c.p. , } \\
& \delta E_{1}\left(0, \epsilon^{\prime}\right) \delta E_{2}\left(0, \epsilon^{\prime}\right)=\left(a^{2}-b^{2}\right) \epsilon_{x x}{ }^{\prime 2}+\left(2 a^{2}+b^{2}\right) \epsilon_{x x}{ }^{\prime} \epsilon_{y y}{ }^{\prime} \\
& -d^{2} \epsilon_{x y}{ }^{2}+\text { c.p. } \\
& \Psi\left(\lambda \hat{k}, \lambda^{\prime} \hat{k}^{\prime}\right)=\frac{1}{2}-\left[D^{2}\left(\hat{k} \cdot \hat{k}^{\prime}\right)^{2}-B^{2}+\left(3 B^{2}-D^{2}\right)\right. \\
& \left.\times\left(\hat{k}_{x}^{2} \hat{k}_{x}^{\prime 2}+\text { c.p. }\right)\right] / 4 E_{\hat{k} \lambda} E_{\hat{k^{\prime}} \lambda^{\prime}} \text { ， }
\end{aligned}
$$

${ }^{22}$ W. Shockley, Phys. Rev. 78, 173 (1950).

${ }^{23}$ H. J. McSkimin, J. Appl. Phys. 24, 988 (1953).

${ }^{24}$ B. N. Brockhouse and P. K. Iyengar, Phys. Rev. 111, 747 (1958).

$$
\begin{gathered}
E_{\hat{k} \lambda}=(-1)^{\lambda}\left[B^{2}+C^{2}\left(\hat{k}_{x}{ }^{2} \hat{k}_{y}{ }^{2}+\text { c.p. }\right)\right]^{1 / 2}, \\
\boldsymbol{\epsilon}_{i j}{ }^{\prime}(\mathbf{q} s)=-\frac{1}{2}\left[q_{i} \hat{e}_{s j}+q_{j} \hat{e}_{s i}\right] .
\end{gathered}
$$

c.p. stands for cyclic permutations of $x, y$, and $z ; a, b$, and $d$ are the usual deformation potentials describing the behavior of the valence-band edge when the crystal is subjected to uniaxial stress. ${ }^{3}$ In Eq. (2.14), the equipartition of phonon energies has been used, since for the phonons interacting with thermal holes $k_{B} T \gg \hbar \omega$. The error introduced in this way is about $2 C_{11} m^{*} / \rho k_{B} T$ $\approx 1 \%$ at $77^{\circ} \mathrm{K}$ for $m^{*}$ equal to the heavy-hole ${ }^{1}$ effective mass $0.3 m$, and $\rho=5.33 \mathrm{~g} \mathrm{~cm}^{-3}$.

We now make the usual approximation of treating the acoustic scattering as elastic, i.e., we neglect $\hbar \omega$ in the energy-conserving $\delta$ function in Eq. (2.14). It is then easy to show that all energy dependence of $P_{\mathrm{ac}}$ is contained in $\delta\left(E^{\prime}-E\right)$. To see this, we observe that (2.15) may be written as

$$
U\left(\lambda \hat{k}, \lambda^{\prime} \hat{k}^{\prime}, \mathbf{q} s\right)=u_{s}\left(\lambda \hat{k}, \lambda^{\prime} \hat{k}^{\prime}, \hat{q}\right) q^{2}
$$

because the "strain" $\epsilon^{\prime} \propto q$. Furthermore, the dispersion-free acoustic spectrum may be expressed as

$$
\hbar \omega_{\mathrm{q} s}=\Omega_{s}(\hat{q}) q .
$$

Inserting of (2.21) and (2.22) in the expression (2.14) for the transition rate yields

$$
\begin{aligned}
P_{\mathrm{ac}}\left(\lambda^{\prime} \mathbf{k}^{\prime}, \lambda \mathbf{k}\right)=\left[(2 \pi)^{3} k_{B} T / V \hbar C_{11}\right] S_{\mathrm{ac}}\left(\lambda \hat{k}, \lambda^{\prime} \hat{k}^{\prime}\right) \\
\\
\times \delta\left(E_{\lambda^{\prime}}\left(\mathbf{k}^{\prime}\right)-E_{\lambda}(\mathbf{k})\right),
\end{aligned}
$$

with

$S_{\mathrm{ac}}\left(\lambda \hat{k}, \lambda^{\prime} \hat{k}^{\prime}\right)=\left[\frac{\hbar^{2} C_{11}}{4 \pi^{2} \rho} \sum_{\mathrm{q} s} u_{s} \Omega_{s}^{-2} \delta_{\mathrm{k}, \mathrm{k}^{\prime}-\mathrm{q}}\right]_{E_{\lambda^{\prime}=E_{\lambda}}}$.

The energy conservation and the parabolic band structure ensure that $S_{\mathrm{ac}}$ does not depend on the magnitude of the $\mathbf{k}$ vectors. This feature is well known from simpler band structures, and leads eventually to the simple temperature dependence of acoustic-scattering limitedtransport parameters.

The introduction of elasticity in the acoustic phonon scattering causes an error of the same order of magnitude as the equipartition approximation, i.e., $1 \%$ at $77^{\circ} \mathrm{K}$. In addition, it is observed that $S_{\mathrm{ac}}$, defined in Eq. (2.24), does not approach a limit as $\hat{k}^{\prime} \rightarrow \hat{k}$ for $\lambda^{\prime}=\lambda$. This is also a consequence of the elasticity approximation, because when $\hat{k}^{\prime} \approx \hat{k}, E_{\lambda}\left(\mathbf{k}^{\prime}\right)-E_{\lambda}(\mathbf{k})$ is no longer large compared to the phonon energy. However, the squared matrix element of the scattering, proportional to $U$, does not particularly favor transitions with $\mathbf{k} \approx \mathbf{k}^{\prime}$, and so the final error is not increased if we avoid sampling the details near $\hat{k}=\hat{k}^{\prime}$ in the numerical treatment of $S_{\mathrm{ac}}$.

\section{Optical Phonon Scattering}

In their theory of optical phonon scattering of holes, Bir and Pikus ${ }^{2}$ have derived the following expression for 
the rate of transitions from state $\mathbf{k}$ in band $\lambda$ to $\mathbf{k}^{\prime}$ in $\lambda^{\prime}$ :

$$
\begin{aligned}
P_{\text {opt }}\left(\lambda^{\prime} \mathbf{k}^{\prime}, \lambda \mathbf{k}\right)= & \frac{\pi}{V \rho \omega_{0} a_{0}^{2}} \sum_{ \pm} \sum_{\mathbf{q} s}\left(n_{0}+\frac{1}{2} \pm \frac{1}{2}\right) U^{0}\left(\lambda \hat{k}, \lambda^{\prime} \hat{k}^{\prime}, \mathbf{q} s\right) \\
& \times \delta_{\mathrm{k}, \mathbf{k}^{\prime} \pm \mathrm{q}} \delta\left(E_{\lambda^{\prime}}\left(\mathbf{k}^{\prime}\right)-E_{\lambda}(\mathbf{k}) \pm \hbar \omega_{0}\right),
\end{aligned}
$$

where $a_{0}=5.66 \AA$ is the lattice constant of Ge introduced in order to give the optical "deformation potential" $d_{0}$ (appearing below), the dimension of energy, and

$$
\begin{gathered}
n_{0}=\left[\exp \left(\hbar \omega_{0} / k_{B} T\right)-1\right]^{-1}, \\
U^{0}\left(\lambda \hat{k}, \lambda^{\prime} \hat{k}^{\prime}, \mathbf{q} s\right)=\delta E_{\lambda}^{0}(\hat{k}) \delta E_{\lambda^{\prime}}{ }^{0}\left(\hat{k}^{\prime}\right)-\delta E_{1}^{0}(0) \\
\times \delta E_{2}{ }^{0}(0) \Psi\left(\lambda \hat{k}, \lambda^{\prime} \hat{k}^{\prime}\right), \\
\delta E_{\lambda}^{0}(\hat{k})=D d_{0}\left(\hat{k}_{x} \hat{k}_{y} \hat{e}_{0 s z}+\text { c.p. }\right) / E_{\hat{k} \lambda} \\
\delta E_{1}{ }^{0}(0) \delta E_{2}{ }^{0}(0)=-d_{0}{ }^{2} .
\end{gathered}
$$

$\Psi$ and $E_{\hat{k} \lambda}$ are defined in Eqs. (2.18) and (2.19).

In Eq. (2.25), we now perform the summation over the three polarization directions $s$ for the optical phonons, and, as pointed out in Sec. II B, we may choose the cubic axes as arbitrary polarization directions. We then get, from (2.27),

$$
\begin{array}{r}
U^{0}\left(\lambda \hat{k}, \lambda^{\prime} \hat{k}^{\prime}\right)=D^{2} d_{0}^{2}\left[\hat{k}_{x} \hat{k}_{y} \hat{k}_{x}{ }^{\prime} \hat{k}_{y}{ }^{\prime}+\text { c.p. }\right] / \\
E_{\hat{k} \lambda} E_{\hat{k}^{\prime} \lambda^{\prime}}+3 d_{0}{ }^{2} \Psi\left(\lambda \hat{k}, \lambda^{\prime} \hat{k}^{\prime}\right) .
\end{array}
$$

The summation of $P_{\text {opt }}$ over $q$ is now straightforward:

$$
\begin{aligned}
P_{\mathrm{opt}}\left(\lambda^{\prime} \mathbf{k}^{\prime}, \lambda \mathbf{k}\right)=\left[(2 \pi)^{3} \omega_{0} / V C_{11}\right] S_{\mathrm{opt}}\left(\lambda^{\prime} \hat{k}^{\prime}, \lambda \hat{k}\right) \\
\quad \times \sum_{ \pm}\left(n_{0}+\frac{1}{2} \pm \frac{1}{2}\right) \delta\left(E_{\lambda^{\prime}}\left(\mathbf{k}^{\prime}\right)-E_{\lambda}(\mathbf{k}) \pm \hbar \omega_{0}\right),
\end{aligned}
$$

where

$$
S_{\text {opt }}\left(\lambda^{\prime} \hat{k}^{\prime}, \lambda \hat{k}\right)=\left(C_{11} / 8 \pi^{2} \rho \omega_{0}^{2} a_{0}^{2}\right) U^{0}\left(\lambda \hat{k}, \lambda^{\prime} \hat{k}^{\prime}\right) .
$$

This form of $P_{\text {opt }}$ is suitable for the treatment of the Boltzmann equation in the following section.

From the expressions for $U^{0}$ and $\Psi$ it is seen that $P_{\text {opt }}$ is invariant under inversions in $\mathbf{k}$ and in $\mathbf{k}^{\prime}$. Thus the optical phonon scattering has the "momentumrandomizing" property which simplifies the later treatment considerably.

\section{BASIS OF CALCULATION}

The calculation of transport parameters from first principles proceeds through three stages: establishment of a fundamental theoretical model as given in Sec. II, solution of the Boltzmann equation to obtain the distribution function, and evaluation of the transport parameters corresponding to the distribution function. In this section we are concerned with the last two stages. The method to be applied is discussed in I and so we shall concentrate on presenting the problem in a form suitable for numerical treatment.

\section{A. Boltzmann Equation}

Let $f_{\lambda}(\mathbf{k})$ be the distribution function of holes in band $\lambda$. Assuming a nondegenerate distribution, the Boltzmann equation governing $f_{\lambda}(\mathbf{k})$ in the steady state reads

$$
\begin{aligned}
q / \hbar\left[\mathbf{F} \cdot \nabla_{\mathbf{k}}-\mathbf{B} \cdot\left(\mathbf{V}_{\lambda}(\mathbf{k}) \times \nabla_{\mathbf{k}}\right)\right] f_{\lambda}(\mathbf{k}) \\
=\sum_{\lambda^{\prime}} \int d^{3} k^{\prime}\left[V /(2 \pi)^{3}\right] \\
\quad \times\left[P\left(\lambda \mathbf{k}, \lambda^{\prime} \mathbf{k}^{\prime}\right) f_{\lambda^{\prime}}\left(\mathbf{k}^{\prime}\right)-P\left(\lambda^{\prime} \mathbf{k}^{\prime}, \lambda \mathbf{k}\right) f_{\lambda}(\mathbf{k})\right],
\end{aligned}
$$

where $\mathbf{F}$ and $\mathbf{B}$ are the applied electric and magnetic fields, $q$ is the charge of a hole, and the velocity

$$
\mathbf{V}_{\lambda}(\mathbf{k})=(1 / \hbar) \nabla_{\mathbf{k}} E_{\lambda}(\mathbf{k})
$$

The total transition rate $P$ is the sum of $P_{\mathrm{ac}}$ from (2.23) and $P_{\text {opt }}$ from (2.31).

According to $\mathrm{I},{ }^{15}$ we then expand the distribution function in a power series in the external fields, and its angular dependence on $\hat{k}, f_{\lambda}(\mathbf{k}) \equiv f_{\lambda}(E, \hat{k})$, is expanded in a suitably chosen orthonormal system of surface harmonics $Y_{n}(\hat{k})$, satisfying

$$
\frac{1}{4 \pi} \int d^{2} \hat{k} Y_{m}(\hat{k}) Y_{n}(\hat{k})=\delta_{m n} .
$$

We then choose the form

$$
f_{\lambda}(E, \hat{k})=f^{0}(x)\left[1+\sum_{n s} \phi_{s n}(\lambda, x) x^{-\frac{1}{2} s} Y_{n}(\hat{k})\right],
$$

where

and

$$
x=E / k_{B} T
$$

$$
f^{0}(x)=c \exp (-x)
$$

is the nondegenerate thermal equilibrium distribution. In accordance with I, $\phi_{s n} \propto F B^{s}$, and the Boltzmann equation is to be solved for these coefficients which, together with (3.4), determine the nonequilibrium distribution function.

In order to transform the transport equation (3.1) into a convenient form, we express the angular dependence of $P\left(\lambda \mathbf{k}, \lambda^{\prime} \mathbf{k}^{\prime}\right), \nabla_{\mathbf{k}} E$, and $\nabla_{\mathbf{k}} E \times \nabla_{\mathbf{k}}$ as expansions in the system $Y_{n}(\hat{k})$. We thus define the matrix elements

$$
\begin{aligned}
& \mathbf{G}_{m 0}(\lambda)=\frac{1}{4 \pi} \int d^{2} \hat{k} Y_{m}(\hat{k}) E_{\lambda}^{-1 / 2} \nabla_{\mathbf{k}} E_{\lambda} Y_{0}(\hat{k}), \\
& \mathbf{H}_{m \mu}(\lambda)=\frac{1}{4 \pi} \int d^{2} \hat{k} Y_{m}(\hat{k}) \nabla_{\mathrm{k}} E_{\lambda} \times \nabla_{\mathbf{k}} Y_{\mu}(\hat{k}) .
\end{aligned}
$$

The transformation of the scattering integral in (3.1) from $\mathbf{k}$ space to $(E, \hat{k})$ space introduces the density-ofstates factor $\frac{1}{2} g_{\lambda}^{-3 / 2}$ with the expansion coefficients

$$
C_{\mu}(\lambda)=\frac{1}{4 \pi} \int d^{2} \hat{k} Y_{\mu}(\hat{k}) \frac{1}{2} g_{\lambda}{ }^{-3 / 2}(\hat{k}) .
$$


For parabolic bands, $\mathbf{G}, \mathbf{H}$, and $C$ are energyindependent.

Similarly, we write for the angular parts of the transition rates

$$
\begin{aligned}
S_{\mathrm{ac}}\left(\lambda \hat{k}, \lambda^{\prime} \hat{k}^{\prime}\right) & =\sum_{\nu \nu^{\prime}} S_{\nu \nu^{\prime}}{ }^{a}\left(\lambda, \lambda^{\prime}\right) Y_{\nu}(\hat{k}) Y_{\nu^{\prime}}\left(\hat{k}^{\prime}\right), \\
S_{\mathrm{opt}}\left(\lambda \hat{k}, \lambda^{\prime} \hat{k}^{\prime}\right) & =\sum_{\nu \nu^{\prime}} S_{\nu \nu^{\prime}}{ }^{0}\left(\lambda, \lambda^{\prime}\right) Y_{\nu}(\hat{k}) Y_{\nu^{\prime}}\left(\hat{k}^{\prime}\right) .
\end{aligned}
$$

As shown in I, the use of the form (3.4) for the distribution function makes it possible to separate different powers of the external fields in the transport equation (3.1). For $s=0$, we find, using the orthogonality properties (3.3) of the functions $Y_{n}(\hat{k})$,

$\frac{q C_{11}}{4 \pi\left(k_{B} T\right)^{2}} \mathbf{F} \cdot \mathbf{G}_{m 0}(\lambda) \cdot 1=\sum_{\lambda^{\prime} n} K_{m n}\left(\lambda, \lambda^{\prime}, x\right) \phi_{0 n}\left(\lambda^{\prime}, x\right)$.

Similarly, the $s=1$ equation reads

$$
\begin{aligned}
\frac{q C_{11}}{4 \pi \hbar\left(k_{B} T\right)^{3 / 2}} \mathbf{B} \cdot \sum_{\mu} \mathbf{H}_{m \mu}(\lambda) \phi_{0 \mu}(\lambda, x) \\
=\sum_{\lambda^{\prime} n} K_{m n}\left(\lambda, \lambda^{\prime}, x\right) \phi_{1 n}\left(\lambda^{\prime}, x\right),
\end{aligned}
$$

and for $s=2$

$$
\begin{aligned}
\frac{q C_{11}}{4 \pi \hbar\left(k_{B} T\right)^{3 / 2}} \mathbf{B} \cdot \sum_{\mu} \mathbf{H}_{m \mu}(\lambda) \phi_{1 \mu}(\lambda, x) \\
=\sum_{\lambda^{\prime} n} K_{m n}\left(\lambda, \lambda^{\prime}, x\right) \phi_{2 n}\left(\lambda^{\prime}, x\right) .
\end{aligned}
$$

The "scattering matrix" $\mathbf{K}$ is readily found to have the form

$$
\begin{gathered}
K_{m n}\left(\lambda, \lambda^{\prime}, x\right)=\delta_{\lambda \lambda^{\prime}} \sum_{\nu} A_{\nu n m} \sum_{\lambda^{\prime \prime} \mu} C_{\mu}\left(\lambda^{\prime \prime}\right)\left[S_{\mu \nu}{ }^{a}\left(\lambda^{\prime \prime}, \lambda\right)\right. \\
+y_{0}\left\{n_{0}\left(1+y_{0} / x\right)^{1 / 2}+\left(n_{0}+1\right)\left(1-y_{0} / x\right)^{1 / 2}\right\} \\
\left.\times S_{\mu \nu}{ }^{0}\left(\lambda^{\prime \prime}, \lambda\right)\right]-\sum_{\mu \nu} A_{\mu \nu n} C_{\mu}\left(\lambda^{\prime}\right) S_{m \nu}{ }^{a}\left(\lambda, \lambda^{\prime}\right),
\end{gathered}
$$

where

$$
\begin{aligned}
A_{\nu n m} & =\frac{1}{4 \pi} \int d^{2} \hat{k} Y_{\nu} Y_{n} Y_{m}, \\
y_{0} & =\hbar \omega_{0} / k_{B} T,
\end{aligned}
$$

and the factor $\left(1-y_{0} / x\right)$ should be replaced by zero if negative. In deriving (3.15) we have used the fact that the optical phonon scattering is momentum-randomizing, and so the "in-scattering" term ${ }^{15}$ vanishes for all odd distribution functions, as is always the case for low-field magnetoconductivity. ${ }^{15}$

The above presentation of the transformation of the transport equation has been given without much explanation because this would take up an unnecessarily large space. Apart from numerical factors, the meaning of the different terms is readily explained by comparison with the principles solution given in I. Thus $\mathbf{G}_{m 0}$ is essentially the matrix element of $\mathbf{F}_{\text {op }}$ between $Y_{m}$ and $Y_{0}=1, \mathbf{H}_{m \mu}$ the matrix element of $\mathbf{B}_{\mathrm{op}}{ }^{15}$ and $K_{m n}$ the matrix element of the scattering operator $S_{\mathrm{op}} \cdot{ }^{15}$ The form of Eqs. (3.12)-(3.14) then follows qualitatively from Eqs. (2.12) and (2.13) of I. Similarly, the form of the scattering matrix element $K_{m n}$ (3.15) is easily interpreted. The first, positive part stands for the "outscattering" term and is diagonal in the band indices $\lambda$ and $\lambda^{\prime}$, whereas the last, negative part is related to the "in-scattering" term. The coefficients $A$ given in Eq. (3.16) are just numerical coupling constants between different $Y$, and have no physical significance.

It is readily seen that the unknown expansion coefficients $\phi_{s n}$ of the distribution function may be obtained by successive solution of the linear, inhomogeneous equations (3.12)-(3.14). It will be made clear in the following section why the particular matrix version of the transport equation derived here is well suited for numerical treatment.

\section{B. Transport Parameters}

The drift velocity is given by

$$
\mathbf{V}_{d}=\frac{\sum_{\lambda} \int d^{3} k f_{\lambda}(\mathbf{k}) \mathbf{V}_{\lambda}(\mathbf{k})}{\sum_{\lambda} \int d^{3} k f_{\lambda}(\mathbf{k})} .
$$

Denoting the carrier density by $n$, we have the current density

$$
\mathbf{j}=n q \mathbf{V}_{d} \text {. }
$$

In cubic crystals like $p$-Ge, the current density may be expressed in the following way to first order in the electric field and to second order in the magnetic field ${ }^{25}$ : $\mathbf{j}=\sigma_{0}\left[\mathbf{F}+\alpha_{B}(\mathbf{F} \times \mathbf{B})\right.$

$$
\left.+\beta_{B} \mathbf{F} B^{2}+\gamma_{B} \mathbf{B}(\mathbf{F} \cdot \mathbf{B})+\delta_{B} \mathbf{T F}\right],
$$

where $\mathbf{T}$ is a diagonal tensor with the elements $B_{x}{ }^{2}, B_{y}{ }^{2}$, and $B_{z}{ }^{2}$. The quantity $\sigma_{0}$ is the zero-field conductivity related to the mobility $\mu_{0}$ by

$$
\sigma_{0}=n q \mu_{0} .
$$

$\alpha_{B}$ is equal to the Hall mobility

$$
\mu_{H}=r \mu_{0},
$$

where $r$ is the so-called Hall factor. Defining the general mobility as

$$
\mu=F^{-1} \hat{F} \cdot V_{d},
$$

we find from (3.19) and (3.20)

$$
\begin{aligned}
\delta \mu / \mu_{0}{ }^{3} B^{2}=\beta_{B}{ }^{\prime}+\gamma_{B}{ }^{\prime}(\hat{F} \cdot \hat{B})^{2} & \\
& +\delta_{B}{ }^{\prime}\left(\hat{F}_{x}{ }^{2} \widehat{B}_{x}{ }^{2}+\hat{F}_{y}{ }^{2} \hat{B}_{y}{ }^{2}+\hat{F}_{z}{ }^{2} \hat{B}_{z}{ }^{2}\right),
\end{aligned}
$$

where $\beta_{B}{ }^{\prime}=\beta_{B} \mu_{0}{ }^{-2}$, etc.

${ }^{25}$ F. Seitz, Phys. Rev. 79, 372 (1950). The notation is the same as employed in Ref. 15. 
In connection with the expansion (3.4) of the distribution function, we write

$$
\mathbf{V}_{d}=\sum_{s} \mathbf{V}_{d s}
$$

where $\mathbf{V}_{d s} \propto F B^{s}$ just as $\phi_{s n}$ in (3.4). We then obtain

$$
\begin{array}{r}
\mathbf{V}_{d s}=2\left(k_{B} T\right)^{1 / 2} \int_{0}^{\infty} d x \exp (-x) x^{1-\frac{1}{2} s} \sum_{\lambda n} \phi_{s n}(\lambda, x) \mathbf{D}_{n}(\lambda) / \\
\pi^{1 / 2} \hbar \sum_{\lambda} C_{0}(\lambda)
\end{array}
$$

where

$$
D_{n}(\lambda)=\frac{1}{4 \pi} \int d^{2} \hat{k} Y_{n}(\hat{k}) \frac{1}{2} g_{\lambda}{ }^{-3 / 2}\left[E_{\lambda}^{-1 / 2} \nabla_{\mathrm{k}} E_{\lambda}\right]
$$

and $C$ was defined in (3.9). From Eqs. (3.23) and (3.25), we now find

and

$$
\mu_{0}=F^{-1} \hat{F} \cdot \mathrm{V}_{d 0},
$$

$$
r=\hat{F} \times \hat{B} \cdot \mathbf{V}_{d 1} / \mu_{0}^{2} F B|\hat{F} \times \hat{B}|,
$$

$$
\delta \mu / \mu_{0}^{3} B^{2}=\hat{F} \cdot \mathbf{V}_{d 2} / \mu_{0}^{3} B^{2} F .
$$

The transport parameters $\mu_{0}, r, \beta_{B}{ }^{\prime}, \gamma_{B}{ }^{\prime}$, and $\delta_{B}{ }^{\prime}$ can thus be calculated from Eqs. (3.24)-(3.30), provided that the distribution function $\phi_{s n}$ is known.

Instead of the reduced magnetoconductivity parameters $\beta_{B}{ }^{\prime}, \gamma_{B}{ }^{\prime}$, and $\delta_{B}{ }^{\prime}$, we shall calculate the corresponding magnetoresistance quantities $b_{B}{ }^{\prime}, c_{B}{ }^{\prime}$, and ${d_{B}}{ }^{26}$ because these are more directly determined by experiment:

$$
\begin{aligned}
& b_{B}{ }^{\prime}=b_{B} \mu_{0}{ }^{-2}=-\beta_{B}{ }^{\prime}-r^{2}, \\
& c_{B}{ }^{\prime}=c_{B} \mu_{0}{ }^{-2}=-\gamma_{B}{ }^{\prime}+r^{2}, \\
& d_{B}{ }^{\prime}=d_{B} \mu_{0}{ }^{-2}=-\delta_{B}{ }^{\prime} .
\end{aligned}
$$

Actually, we rather calculate the transverse magnetoresistance coefficient $b_{B}{ }^{\prime}$, the longitudinal coefficient for fields in the $\langle 110\rangle$ direction $b_{B}{ }^{\prime}+c_{B}{ }^{\prime}+\frac{1}{2} d_{B}{ }^{\prime}$, and the longitudinal coefficient for the $\langle 100\rangle$ directions $b_{B}{ }^{\prime}+c_{B}{ }^{\prime}$ $+d_{B}{ }^{\prime}$. We shall later see that these three combinations represent different symmetry parts of the distribution function. ${ }^{15}$ From the discussion in I, it is therefore expected that they will be of different orders of magnitude.

It is easily seen from the temperature and energy dependence of the acoustic-phonon-scattering rates and from a qualitative solution of the Boltzmann equations, (3.12)-(3.14), that for pure acoustic scattering $\mu_{0} \propto T^{-3 / 2}$ and $r, b_{B}{ }^{\prime}, c_{B}{ }^{\prime}$, and $d_{B}{ }^{\prime}$ are independent of temperature. Deviations from this simple temperature dependence are caused by optical phonon scattering and nonparabolicity, the effect of the latter being included below.

\section{Inclusion of Nonparabolicity}

We now proceed to incorporate the slight nonparabolicity of the light hole band as expressed in the approximate formula (2.8). Only the direct band-structure

\footnotetext{
${ }^{26}$ G. L. Pearson and H. Suhl, Phys. Rev. 83, 768 (1951).
}

effects will be considered, and so the scattering is assumed to be unaffected. The corresponding error in the transport parameters is difficult to estimate, but is probably of second order, since it vanishes for a particular choice of the deformation potentials, i.e., $b=d=0$ (exact only for isotropic acoustic phonon spectrum ${ }^{4}$ ).

In the above approximation, the effects of nonparabolicity is a slight energy dependence of the coefficients $C, \mathbf{D}, \mathbf{G}$, and $\mathbf{H}$. Thus, for $\lambda=2$,

$$
\begin{aligned}
& C \rightarrow C\left[1+\frac{5}{2} C_{2} E / \Delta\right], \\
& \mathbf{D} \rightarrow \mathbf{D}\left[1+C_{2} E / \Delta\right], \\
& \mathbf{G} \rightarrow \mathbf{G}\left[1-\frac{3}{2} C_{2} E / \Delta\right], \\
& \mathbf{H} \rightarrow \mathbf{H}\left[1-2 C_{2} E / \Delta\right] .
\end{aligned}
$$

Finally, in the formula (3.26) for $\mathrm{V}_{d s}$, the sum $\sum_{\lambda} C_{0}(\lambda)$ must be replaced by an integral

$$
\sum_{\lambda} C_{0}(\lambda) \rightarrow 2 \pi^{-1 / 2} \int_{0}^{\infty} d x x^{-1 / 2} \exp (-x) \sum_{\lambda} C_{0}(\lambda)
$$

where $C_{0}(2)$ now includes the nonparabolicity correction to $C$ as expressed in (3.32).

\section{METHOD OF COMPUTATION}

\section{A. Consequences of Cubic Symmetry}

In the treatment of the Boltzmann equation in Sec. III A, we did not make use of the inherent cubic symmetry of the problem. As shown in I, important simplifications follow from the full application of the symmetry. The only condition to be fulfilled is that the system $Y_{n}(\hat{k})$ should be chosen as the cubic harmonics, ${ }^{27}$ which are classified according to the irreducible representations and rows of the full cubic point group $\Gamma$. It then follows from I and Eq. (3.4) that the distribution function coefficients $\phi_{s n}$ are classified in the same way, and the calculation of $\mu_{0}, r, \beta_{B}{ }^{\prime}, \gamma_{B}{ }^{\prime}$, and $\delta_{B}{ }^{\prime}$ may proceed as in Sec. III A of I without reference to a specific field configuration. The procedure for obtaining $\phi_{0 n}, \phi_{1 n}$, and $\phi_{2 n}$ is shown schematically in Fig. 1. The notation for the irreducible representations and the corresponding parts of the distribution function is the same as in I. Then

$$
\begin{aligned}
\mu_{0} \sim \delta_{01}, \\
r \sim \delta_{13}, \\
\beta_{B}{ }^{\prime} \sim-\delta_{23}+\delta_{24}, \\
\beta_{B}{ }^{\prime}+\gamma_{B}{ }^{\prime}+\frac{1}{2}{\delta_{B}}^{\prime} \sim \delta_{24}+\frac{1}{4} \delta_{22}+\delta_{21}, \\
\beta_{B}{ }^{\prime}+\gamma_{B}{ }^{\prime}+\delta_{B}{ }^{\prime} \sim \delta_{22}+\delta_{21},
\end{aligned}
$$

where the sign $\sim$ denotes "derived from."

For the cubic harmonic ${ }^{27}$ belonging to the same irreducible representation and row, the order indicates

${ }^{27}$ F. C. von der Lage and H. Bethe, Phys. Rev. 71, 612 (1947). 
the degree of anisotropy. Since the deviation from isotropy of the energy spectrum and of the phonon scattering rates is not extreme in $p$-Ge, we may estimate the order-of-magnitude relation between $\delta_{21}, \delta_{22}, \delta_{23}$, and $\delta_{24}$ from a consideration of their symmetry origins in $\phi_{1}: \Gamma_{1}{ }^{\prime}, \Gamma_{12}{ }^{\prime}, \Gamma_{15}$, and $\Gamma_{25}$, respectively. The lowest order of cubic harmonics for these representations is ${ }^{27} 9,5,1$, and 3, respectively. From the simple anisotropy argument we then expect $\left|\delta_{23}\right| \gg\left|\delta_{24}\right| \gg\left|\delta_{22}\right| \gg\left|\delta_{21}\right|$, and so

$$
\left|\beta_{B}{ }^{\prime}\right| \gg\left|\beta_{B}{ }^{\prime}+\gamma_{B}{ }^{\prime}+\frac{1}{2} \delta_{B}{ }^{\prime}\right| \gg\left|\beta_{B}{ }^{\prime}+\gamma_{B}{ }^{\prime}+\delta_{B}{ }^{\prime}\right| \text {. }
$$

A similar relation holds between the corresponding magnetoresistance coefficients. Our results confirm this estimate.

Another important consequence of cubic symmetry as discussed in $I$ is that the expansion coefficients $S_{\nu \nu^{\prime}}\left(\lambda, \lambda^{\prime}\right)$ of the scattering rates defined in Eqs. (3.10) and (3.11) are diagonal in the irreducible representations and rows therein. This reduces the number of distinct coefficients drastically. An additional result obtained in I for the low-field magnetoconductivity problem is that we require only the $\Gamma_{1}, \Gamma_{1}{ }^{\prime}, \Gamma_{12}{ }^{\prime}, \Gamma_{15}$, and $\Gamma_{25}$ coefficients of $S_{\mathrm{ac}}$ and the $\Gamma_{1}$ coefficients of $S_{\text {opt }}$. Owing to the relatively simple angular dependence of $S_{\text {opt }}$, it is easy to find the $\Gamma_{1}$ part by inspection of Eqs. (2.30) and (2.32):

$$
S_{\mathrm{opt}}\left(\Gamma_{1}\right)=\frac{C_{11}}{8 \pi^{2} \rho \omega_{0}^{2} a_{0}^{2}} \times \frac{3}{2} d_{0}^{2} .
$$

The remaining part of $S_{\text {opt }}$ transforms according to $\Gamma_{12}$ and $\Gamma_{25}{ }^{\prime}$ and has no influence on low-electric-field magnetoconductivity. The expansion coefficients of (4.7) reduce to $\left(Y_{0}=1\right)$

$$
S_{\nu \nu^{\prime}} 0\left(\lambda, \lambda^{\prime}\right)=S_{\text {opt }}\left(\Gamma_{1}\right) \delta_{\nu 0} \delta_{\nu^{\prime} 0}
$$

Thus, for our present purpose, optical phonon scattering is isotropic.

\section{B. Important Details of Computation}

It is not our intention to describe the actual calculations in detail. For instance, a table giving all the

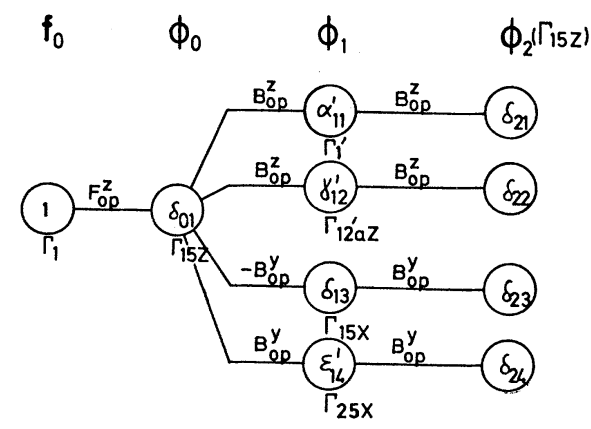

FIG. 1. Schematic illustration of the procedure for calculation of the various parts of the distribution function. The diagram follows from Ref. 15 and the notation is the same except for $\phi_{0,}$ $\phi_{1}$, and $\phi_{2}$.

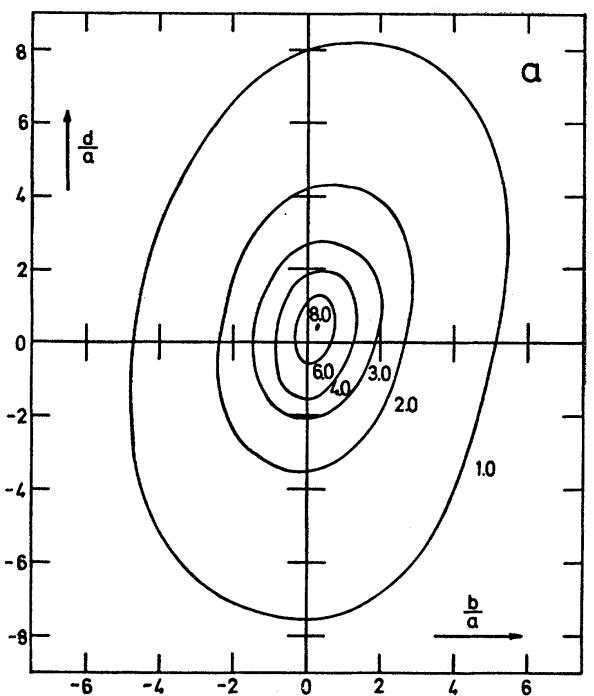

FIG. 2. Contour diagram of the deformation potential $a$ adjusted to give the experimental acoustic mobility.

necessary coupling coefficients $A_{\nu n m}$, defined in Eq. (3.16), has been omitted although these have not been published before. Nevertheless, we feel that some details may be important in order to establish the reliability of the results.

For a practical application, the infinite-series expansions in the system $Y_{n}(\hat{k})$ or similar infinite matrices introduced in Sec. III in the transformation of the Boltzmann equation must necessarily be truncated in order to deal with a finite number of coefficients. Since the coefficients $C, \mathbf{G}, \mathbf{H}$, and $\mathbf{D}$ are single surface integrals over the unit sphere, they may be computed with a desired accuracy by direct numerical integration within a reasonable time. They depend only upon the energy spectrum of holes, and so the truncation of the corresponding series depends only on the anisotropy of the energy spectrum. To achieve an over-all accuracy better than $1 \%$ for holes in Ge, it has been found necessary to include terms up to eighth order in the cubic harmonics. This is mostly dictated by the small longitudinal magnetoresistance parameter $\left(b_{B}{ }^{\prime}+c_{B}{ }^{\prime}+d_{B}{ }^{\prime}\right)$. According to the discussion in Sec. III A, the second-order distribution function $\delta_{21}$ originates from the $\Gamma_{1}{ }^{\prime}$ representation (in first order of the magnetic field) where the lowest order of harmonics is 9 . It follows that $\delta_{21}$ falls outside the accuracy of the calculation, and because it does not lead to a separate transport parameter, $\delta_{21}$ (and so $\alpha_{11}{ }^{\prime}$ in $\phi_{1}$ ) may be neglected entirely.

The truncation of the double-series expansion (3.10) of the acoustic scattering rate is determined by the anisotropy of the scattering which actually depends very little on the anisotropy of the hole energy spectrum. The expansion coefficients $S_{\nu \nu^{\prime}}{ }^{a}$ are computed by interpolation in $\left(\lambda \hat{k}, \lambda^{\prime} \hat{k}^{\prime}\right)$ space with a series having the maximum total order $\nu+\nu^{\prime}=8$. After that the $\nu+\nu^{\prime}=8$ terms are cut off, and the remaining terms with $\nu+\nu^{\prime} \leqslant 6$ result in 
a maximum over-all $1 \%$ sensitivity of the transport parameters to variations in the choice of interpolation points. It is our belief that this procedure is more relevant than the least-squares method used by Tiersten. ${ }^{7}$ However, the small differences between our coefficients and those found by Tiersten ${ }^{7}$ using $\nu+\nu^{\prime} \leqslant 4$ may perhaps by ascribed to the fact that, in our method, the various symmetry parts of $S_{\mathrm{ac}}$ are separated before the comparison with the truncated series. This is not possible in Tiersten's case, due to the simple choice of interpolation points in the main symmetry directions. ${ }^{7}$ As shown below, the principal anisotropy of the acoustic scattering is connected with the even representations $\Gamma_{12}$ and $\Gamma_{25}{ }^{\prime}$ because all the magnetoconductivity results may be interpreted in terms of almost isotropic relaxation times. This conclusion was also reached by Tiersten ${ }^{7}$ for a special choice of deformation potentials. The anisotropy of the acoustic phonon spectrum has practically no influence on the results. ${ }^{4}$ The effect is drowned in anisotropies already present from other sources.

\section{Previous Theoretical Results}

For special choices of the deformation potentials $a$, $b$, and $d$, the results of our calculation may be compared with earlier theoretical calculations pertaining to pure acoustic scattering. Using Tiersten's parameters ${ }^{7}$ $a=-2.0 \mathrm{eV}, b=2.1 \mathrm{eV}$, and $d=7.0 \mathrm{eV}$, we calculate a mobility which deviates only $2 \%$ from his result. For $b=d=0$, we may compare our result with the results of Lax and Mavroides, ${ }^{6}$ inserting their values of the inverse mass parameters and using an isotropic acoustic spectrum, although none of these changes is found to be important. The Hall factor of 2.07 coincides with our result, but the magnetoconductivity parameters show

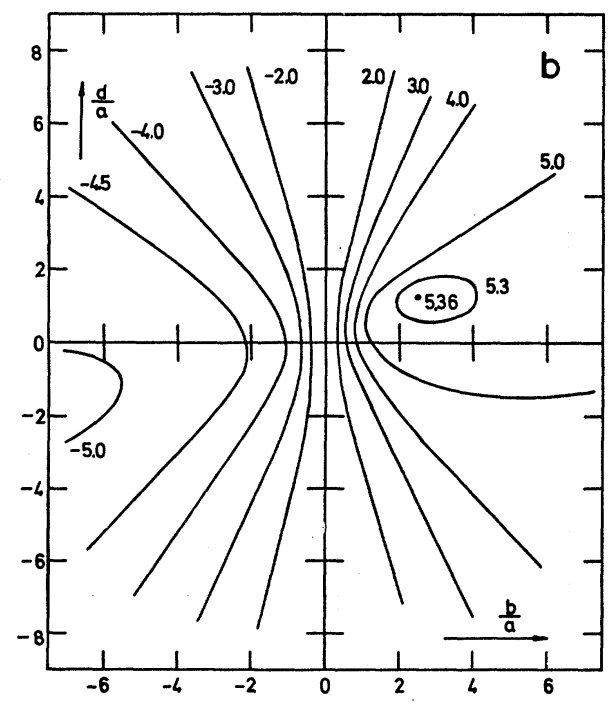

FIG. 3. Contour diagram of the deformation potential $b$ adjusted to give the experimental acoustic mobility.

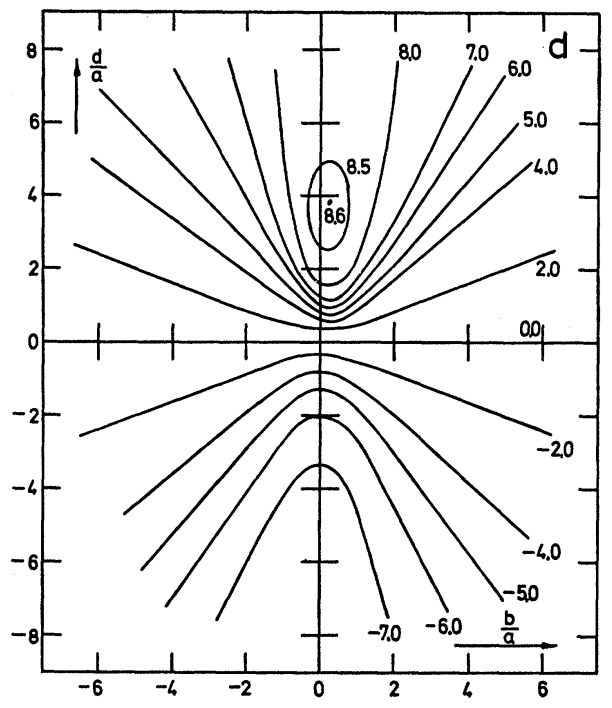

FIG. 4. Contour diagram of the deformation potential $d$ adjusted to give the experimental acoustic mobility.

significant deviations which seem to be due to a series of small errors in Lax and Mavroides's paper. ${ }^{28}$

\section{RESULTS}

\section{A. Pure Acoustic Phonon Scattering}

For pure acoustic scattering, the transition rate has a quadratic form in the deformation potentials $a, b$, and $d$. For constant ratios $b / a$ and $d / a$, the mobility $\mu_{0}$ will

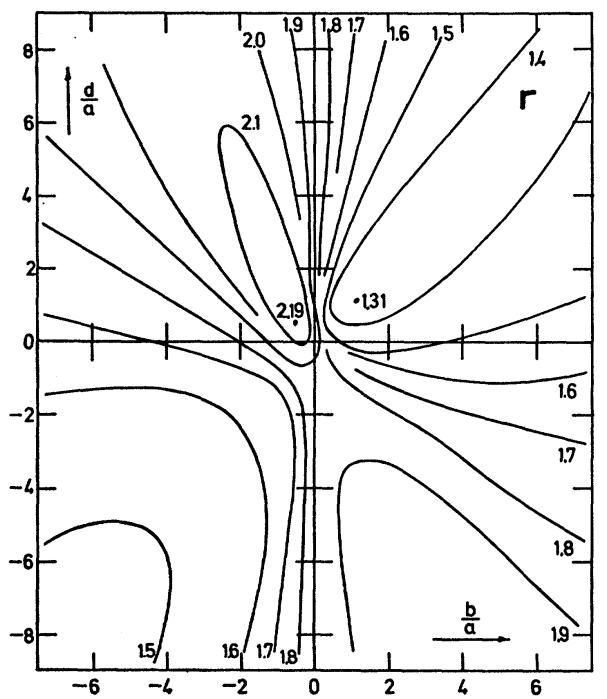

FIG. 5. Contour diagram of the Hall factor $r$ for pure acoustic scattering.

${ }^{28}$ In addition to the published error (Ref. 6), Eq. (5) in the second paper of Ref. 6 contains three errors: (1) $\sigma_{x y y x}$ and $\sigma_{x y x y}$ should be interchanged, (2) the first and third series should be multiplied by 2 , and (3) in the series for $\sigma_{x x y y}$, the coefficient of $\gamma^{2}$ is incorrect as far as we can estimate from our results; we have not attempted to repeat the calculations of Ref. 6. 


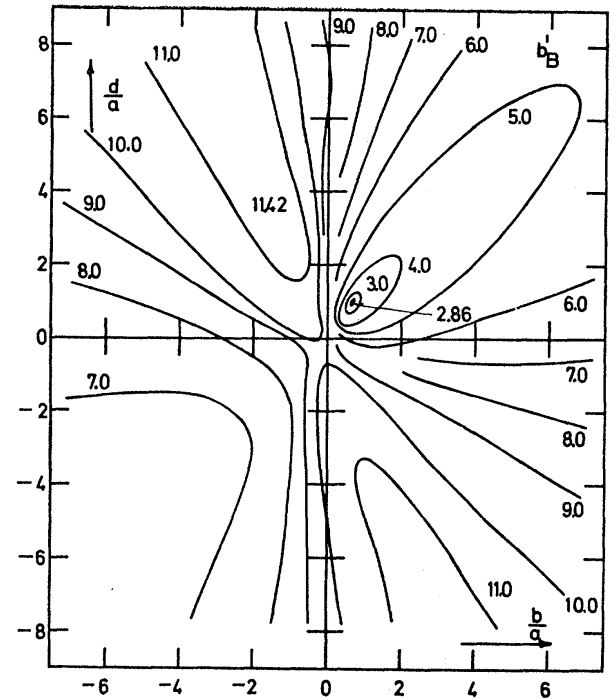

FIG. 6. Contour diagram of the transverse magnetoresistance coefficient $b_{B}{ }^{\prime}$ for pure acoustic scattering.

be inversely proportional to $a^{2}$, whereas the Hall factor $r$ and the reduced magnetoresistance $\delta \mu / \mu_{0}^{3} B^{2}$ are independent of $a$. It follows that we may insert $a^{\prime}=1$, $b^{\prime}=b / a$, and $d^{\prime}=d / a$, calculate the corresponding mobility $\mu^{\prime}$, and finally scale the deformation potentials so that the experimental acoustic mobility ${ }^{1,12} \mu_{0}=3.37$ $\times 10^{7}\left(T / 1^{\circ} \mathrm{K}\right)^{-1.5} \mathrm{~cm}^{2} / \mathrm{V} \mathrm{sec}$ is obtained.

$$
(a, b, d)=\left(\mu^{\prime} / \mu_{0}\right)^{1 / 2}\left(a^{\prime}, b^{\prime}, d^{\prime}\right) \text {. }
$$

The value of $r$ and $\delta \mu / \mu_{0}^{3} B^{2}$ is unaffected by this procedure. Then $a, b, d, r, b_{B}{ }^{\prime},\left(b_{B}{ }^{\prime}+c_{B}{ }^{\prime}+\frac{1}{2} d_{B}{ }^{\prime}\right)$, and $\left(b_{B}{ }^{\prime}+c_{B}{ }^{\prime}+d_{B}{ }^{\prime}\right)$ are functions of $b / a$ and $d / a$ only, and are shown in Figs. 2-8. In Sec. II, we defined the sign of $a, b$, and $d$ as corresponding to hole energies, but in the present problem, the relevant sign of $b$ and $d$ is rather the sign of $B b$ and $D d$, since we have chosen $B$ and $D$ positive in Eqs. (2.3) and (2.6). It is evident from the quadratic form of the transition rates that the total sign of $a, B b /|B|$, and $D d /|D|$ is irrelevant for transport calculations. Therefore, we have chosen $a$ to be positive in Figs. 2-4 without loss of generality. However, it is known from several experiments on uniaxially strained $\mathrm{Ge}^{29,30}$ that $B b$ and $D d$ are positive and this fixes the absolute sign.

The results presented for pure acoustic scattering in Figs. 2-8 may be interpreted by a relatively simple model of the scattering. This model is suggested by the elliptical form of the curves of constant $a$ in Fig. 2. If we take $B=D=0$ in Eq. (2.15) and the following equations and assume an isotropic phonon spectrum characterized by the average longitudinal and transverse elastic constants

$$
\begin{aligned}
& C_{l}=\frac{1}{5}\left(3 C_{11}+2 C_{12}+4 C_{44}\right), \\
& C_{t}=\frac{1}{5}\left(C_{11}-C_{12}+3 C_{44}\right),
\end{aligned}
$$

${ }^{29}$ J. J. Hall, Phys. Rev. 128, 68 (1962).

${ }^{30}$ I. Balslev, Phys. Rev. 143, 636 (1966). we obtain an effective deformation potential $\Xi_{\text {eff }}$ of the form

$$
\Xi_{\mathrm{eff}}^{2}=a^{2}+\left(C_{l} / C_{t}\right)\left(b^{2}+\frac{1}{2} d^{2}\right) .
$$

In this model, the curve of constant $a$ is given by

$$
b^{\prime 2}+\frac{1}{2} d^{\prime 2}=\left(C_{l} / C_{t}\right)\left(\Xi_{\mathrm{eff}}^{2} / a^{2}-1\right) .
$$

Apart from the shift of the center and the rotation of the principal axes, the ellipses in Fig. 2 are quantitatively consistent with Eq. (5.4). The shift and the rotation can be explained by $B \neq 0 \neq D$. Remaining in the simple picture, we may assume effective deformation potentials which are different for the two bands, and are of the form

$$
\begin{aligned}
\Xi_{\lambda, \mathrm{eff}}^{2}=a^{2}\left[1+\left(C_{l} / C_{t}\right)\right. & \left(b^{\prime 2}+\frac{1}{2} d^{\prime 2}\right) \\
& \left.+(-1)^{\lambda}\left(\xi b^{\prime}+\eta d^{\prime}+\zeta b^{\prime} d^{\prime}\right)\right],
\end{aligned}
$$

where $\xi, \eta$, and $\zeta$ are parameters determined from the orientation and center of one of the ellipses in Fig. 2, and they are found to be small compared to 1. Equation (5.5) corresponds to simple, isotropic relaxation times in the two bands, and this model is found to fit even the details of Figs. 2-8 to within 5\% accuracy. The factors depending on band structure are determined from Lax and Mavroides's theory ${ }^{6,28}$ for $b^{\prime}=d^{\prime}=0$.

The results will be discussed further when we compare them with experimental data. Finally, we want to mention that a change in the mass parameters $A, B$, and $C$ within the uncertainty intervals, shown in Eq. (2.3), has very little effect $(<1 \%)$ on the transport parameters.

\section{B. Mixed Phonon Scattering and Nonparabolicity}

The temperature dependence of the low-field transport parameters has been calculated including optical phonon scattering and nonparabolicity. The optical

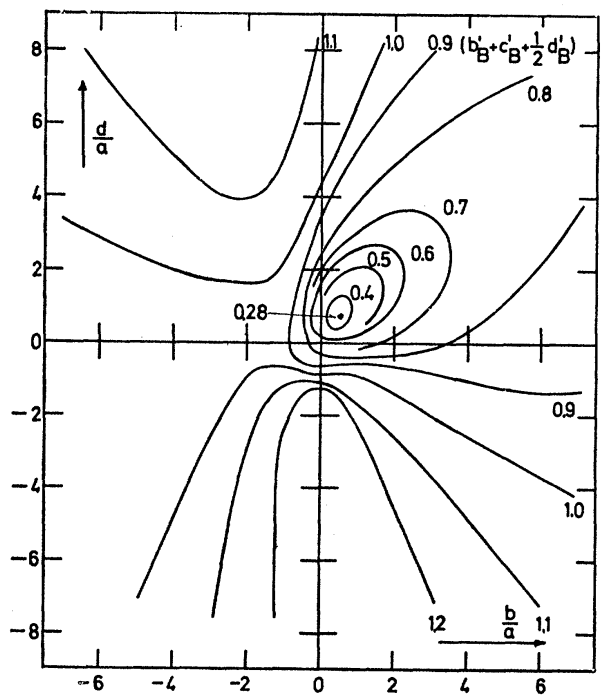

FIG. 7. Contour diagram of the longitudinal magnetoresistance coefficient $\left(b_{B}{ }^{\prime}+c_{B}{ }^{\prime}+\frac{1}{2} d_{B}{ }^{\prime}\right)$ for pure acoustic scattering. 
deformation potential $d_{0}$ is determined so that the mobility calculated at $T=300^{\circ} \mathrm{K}$ coincides with the experimental value ${ }^{12} \mu_{300}=2060 \mathrm{~cm}^{2} / \mathrm{V}$ sec. This automatic adjustment of $d_{0}$ is performed by the computer using an iterative procedure based on the simple interpolation formula

$$
1 / \mu=1 / \mu_{\mathrm{ac}}+p d_{0}{ }^{2},
$$

where $p$ is a constant varied from step to step. The fact that three iterations (starting with $d_{0}=10 \mathrm{eV}$ ) sufficed for an accuracy better than $1 \%$ supports the approximate validity of Eq. (5.6) which is a direct consequence of the relaxation time interpretation of the acoustic scattering given above. This simple picture is further substantiated by the feature that the adjusted value of $d_{0}$ is independent of the initial choice of $b / a$ and $d / a$. The actual variation of $d_{0}$ is within $1 \%$, which is of the same order of magnitude as the estimated maximum error in the mobility calculation for mixed lattice scattering. Another feature is that the temperature dependence of the Hall factor and the magnetoresistance is found to depend only upon the values obtained for pure acoustic scattering, not explicitly on the ratios $b / a$ and $d / a$.

The fitted value of $d_{0}$ is $d_{0}=(6.41 \pm 0.03) \mathrm{eV}$. If nonparabolicity is neglected, $d_{0}=6.53 \mathrm{eV}$, so that the influence of nonparabolicity on the mobility at $300^{\circ} \mathrm{K}$ is about $4 \%$. Effects of the same order of magnitude are found for the galvanomagnetic effects, though somewhat larger. The smallness of the nonparabolicity contribution justifies the approximate treatment of this phenomenon in Sec. II A.

The temperature dependence of the mobility calculated as described above is shown in Fig. 9. The $T^{-2.3}$ dependence in the range $120-300^{\circ} \mathrm{K}$ found experimentally ${ }^{1,12}$ is clearly reproduced and is thus a property

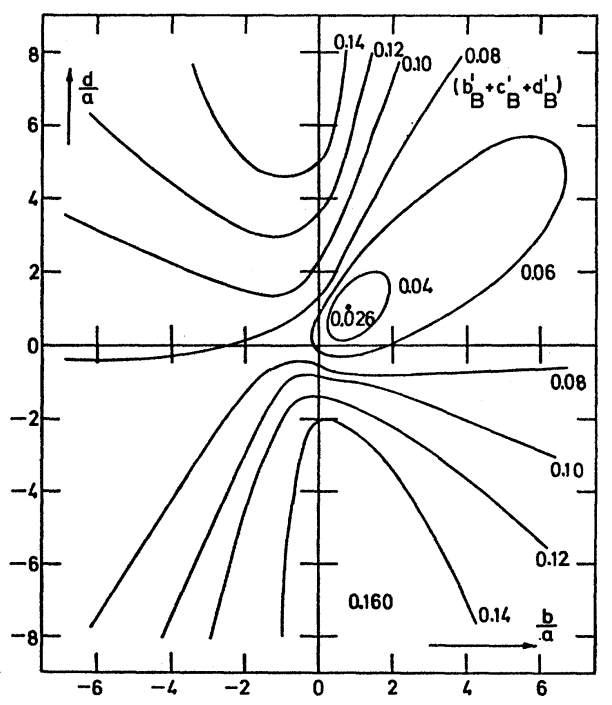

FIG. 8. Contour diagram of the longitudinal magnetoresistance coefficient $\left(b_{B}{ }^{\prime}+c_{B}{ }^{\prime}+d_{B}{ }^{\prime}\right)$ for pure acoustic scattering.

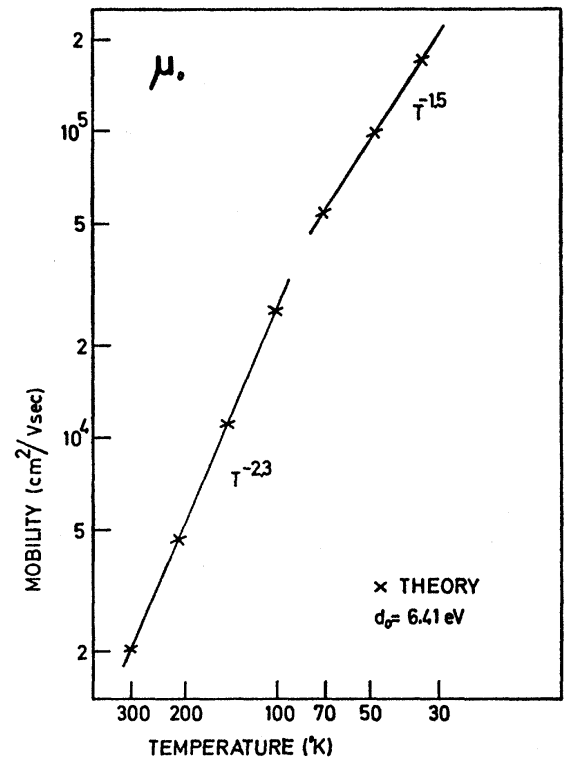

FIG. 9. Temperature dependence of mobility $\mu_{0}$ calculated with mixed phonon scattering and nonparabolicity. $d_{0}=6.41 \mathrm{eV}$.

of the relatively strong optical phonon scattering. ${ }^{1}$ The temperature dependence of the Hall factor and of the magnetoresistance is shown in Figs. 10-13 for selected values of the low-temperature, pure-acoustic-scattering parameters. It is observed that the inclusion of optical phonon scattering in general raises the value of $r, b_{B}{ }^{\prime}$, $\left(b_{B}{ }^{\prime}+c_{B}{ }^{\prime}+\frac{1}{2} d_{B}{ }^{\prime}\right)$, and $\left(b_{B}{ }^{\prime}+c_{B}{ }^{\prime}+d_{B}{ }^{\prime}\right)$. This is due to the strong energy dependence of this type of scattering. It is worth noting that, whereas the mobility is unaffected by optical phonon scattering below $77^{\circ} \mathrm{K}$, the galvanomagnetic effects show some dependence even down to $50^{\circ} \mathrm{K}$.

\section{COMPARISON WITH EXPERIMENT}

One of the attractive features of the deformationpotential theory of acoustic scattering is that its param-

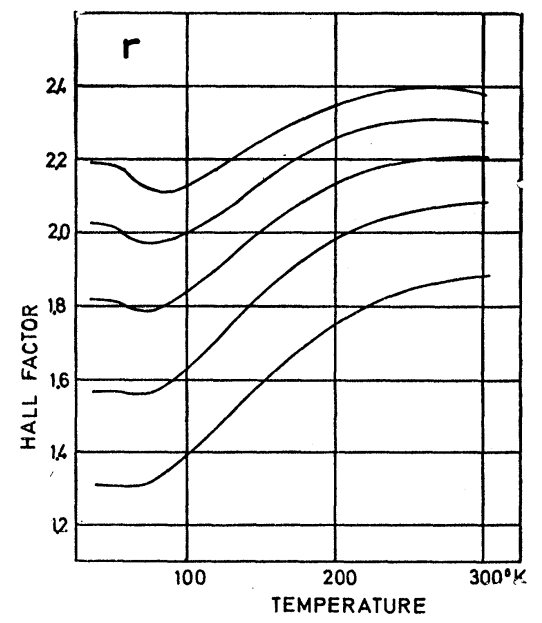

FIG. 10. Temperature dependence of the Hall factor $r$ for representative values of the low-temperature limit. $d_{0}=6.41 \mathrm{eV}$. 


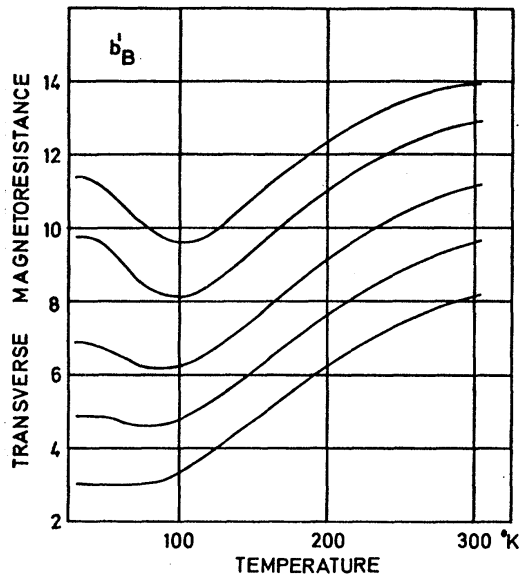

FIg. 11. Temperature dependence of transverse magnetoresistance $b_{B}{ }^{\prime}$ for representative values of the low-temperature limit. $d_{0}=6.41 \mathrm{eV}$.

eters, the deformation potentials $a, b$, and $d$, may be determined from experiments which are independent of acoustic scattering of holes. Thus, the only adjustable parameter in the theory is $d_{0}$, and in view of the reliability of the value of the mobility, ${ }^{1,12}$ it follows from our theory that the fitted value of $d_{0}=6.41 \mathrm{eV}$ is beyond question if the fundamental theoretical model is correct. On the other hand, the theory for pure acoustic scattering contains no adjustable parameters, and a comparison with relevant experimental data therefore concludes in a judgment of deformation-potential theory.

\section{A. Deformation Potentials}

When the crystal is subjected to uniaxial stress, the deformation potentials $b$ and $d$ determine the splitting of the valence-band degeneracy at $\mathbf{k}=0$ and the change in the form of the band structure in the vicinity of this point, whereas $a$ determines the total shift of the valenceband edge. ${ }^{3}$ Thus, $b$ and $d$ are connected directly with the valence band, while $a$ can only be found in combination with the corresponding parameter for the conduction band $\boldsymbol{\Xi}_{d}+\frac{1}{3} \Xi_{u}{ }^{1}$.

The parameters $b$ and $d$ have been determined with appreciable accuracy by several experimental methods, and the most reliable results are reviewed in Table I. It is evident that the values obtained for $d$ differ significantly in the following three groups of methods: (1) direct optical edge, (2) indirect optical edge, and (3) other experiments not involving interband transitions. This is a first indication that the present version of deformation-potential theory may not be entirely correct. Since acoustic phonon scattering is an intraband process, we shall take the values of $b$ and $d$ from the third group as relevant for comparison with the transport results of the present work. Thus $|b| \approx 2.1 \mathrm{eV}$ and $|d| \approx 6.5 \mathrm{eV}$ will be used, but, as we shall see, this choice turns out to be unimportant because the comparison with experimental transport parameters below

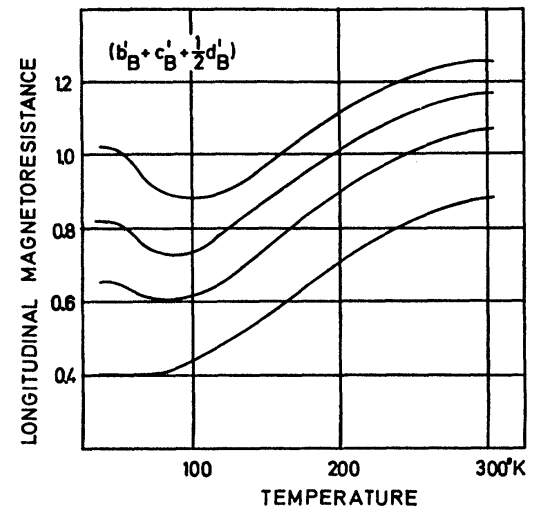

FIG. 12. Temperature dependence of longitudinal magnetoresistance $\left(b_{B}{ }^{\prime}+c_{B}{ }^{\prime}+\frac{1}{2} d_{B}{ }^{\prime}\right)$ for representative values of the lowtemperature limit. $d_{0}=6.41 \mathrm{eV}$.

indicates that a more fundamental inconsistency may exist.

A value of $a$ may be found from the band-gap dilatational coefficient $E_{1 g}$ (in electron energy):

$$
E_{1 g}=\Xi_{d}+\frac{1}{3} \Xi_{u}+a
$$

together with a conduction-band transport determination of $\Xi_{d}+\frac{1}{3} \Xi_{u}$. Information on $E_{1 g}$ can be obtained from studies of changes in the optical indirect interband transition with hydrostatic pressure ${ }^{31}$ and uniaxial stress. ${ }^{30,32}$ Thus Paul finds ${ }^{31} E_{1_{g}}=-3.8 \mathrm{eV}$, while Balslev obtains the value ${ }^{32} E_{1 g}=-2.9 \mathrm{eV}$. Similarly, the published values of $\Xi_{d}+\frac{1}{3} \Xi_{u}$ show quite an appreciable spread because $\boldsymbol{\Xi}_{u}$ is so large $(18 \mathrm{eV})$ and the ratio $\Xi_{d} / \Xi_{u}$ is close to $-\frac{1}{3} .{ }^{1}$ The range of values of $a$ determined from Eq. (6.1) is approximately -1.2 to -3.6 $\mathrm{eV}$, but the most important result of the above discus-

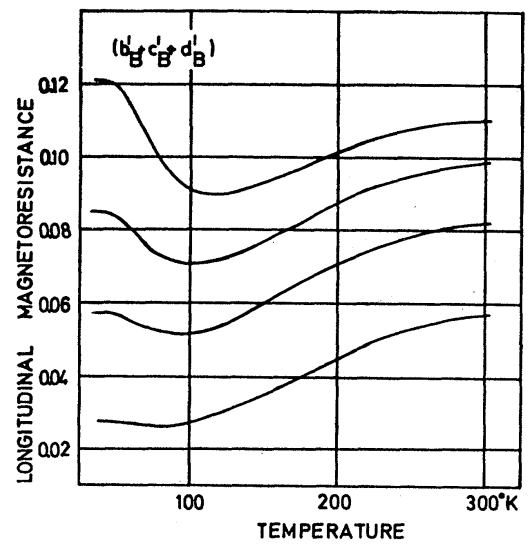

Fig. 13. Temperature dependence of longitudinal magnetoresistance $\left(b_{B}{ }^{\prime}+c_{B}{ }^{\prime}+d_{B}{ }^{\prime}\right)$ for representative values of the low-temperature limit. $d_{0}=6.41 \mathrm{eV}$.

${ }^{31}$ W. Paul, J. Appl. Phys. 32, 2082 (1961); W. Paul and H. Brooks, in Progress in Semiconductors, edited by A. F. Gibson (Heywood and Co., Ltd., London, 1961), Vol. 7, p. 135.

${ }^{32}$ I. Balslev, Phys. Letters 24A, 113 (1967). 
TABLE I. Deformation potentials $b$ and $d$ determined from experiments on strained Ge.

\begin{tabular}{cc}
\hline \hline$b \mid(\mathrm{eV})$ & $|d|(\mathrm{eV})$ \\
\hline $2.7 \pm 0.3$ & $4.7 \pm 0.5^{\mathrm{a}}$ \\
$2.4 \pm 0.2$ & $4.1 \pm 0.4^{\mathrm{b}}$ \\
$1.8 \pm 0.3$ & $3.7 \pm 0.4^{\mathrm{e}}$ \\
$2.4 \pm 0.4$ & $3.5 \pm 0.4^{\mathrm{d}}$ \\
$2.1 \pm 0.2$ & $7.0 \pm 1.5^{\mathrm{e}}$ \\
& $6.0 \pm 0.6^{\mathrm{f}}$ \\
\hline
\end{tabular}

a Direct optical edge splitting, dc method, absorption. See A. M. Glass Can. J. Phys. 43, 12 (1965).

b Direct optical edge splitting, stress modulation, reflection. See I. Balslev, Solid State Commun. 5, 315 (1967).

Indirect optical edge splitting, energy modulation, absorption. See Ref

30 .

Acceptor binding-energy variation. See Ref. 29

f Cyclotron resonance in the quantum limit. See J. C. Hensel, Solid State Commun. 4, 231 (1966).

sion is that $a$ is certainly negative, i.e., it has the opposite sign of $B b$ and $D d$.

\section{B. Transport Properties}

The experimentally data on mobility ${ }^{1,12}$ have already been used in the theory to adjust $a, b, d$, and $d_{0}$ for fixed ratios $b / a$ and $d / a$. These adjustments can always be made, and the experimental temperature dependence $T^{-2.3}$ between 120 and $300^{\circ} \mathrm{K}$ is realized for any choice of $b / a$ and $d / a$. Thus, the mobility alone is not a good indicator of the actual values of $b / a$ and $d / a$. As found by Tiersten, ${ }^{7}$ the set $(a, b, d)=(-1.92,2.01,6.71) \mathrm{eV}$ fits the mobility and is consistent with the results of experiments on strained $p$-Ge discussed above. It is, however, seen from Figs. $2-4$ that with $|b| \approx 2 \mathrm{eV}$ and $|d| \approx 7 \mathrm{eV}$ the determination of $a$ from the mobility is very uncertain: $a=-1.5 \pm 1.5 \mathrm{eV}$.

Figure 14 shows experimental data on the Hall factor selected to represent pure lattice scattering and the lowmagnetic-field limit. ${ }^{8-10,33,34}$ This limit is determined by the conduction $(\mu B)^{2} \ll 1$, where $\mu$ is the light hole mobility. Thus, at $77^{\circ} \mathrm{K}$, magnetic fields below $100 \mathrm{G}$ are needed ${ }^{10}$ and only the results published by Beer and Willardson ${ }^{10}$ fulfill this condition. From a simplified theory of the dependence of $r$ on the magnetic field,,$^{10}$ the low-field limit should be the maximum value of $r$. It is seen in Fig. 14 that many experimental points lie above those taken from Ref. 10, and it follows that it is rather impossible to draw a definite conclusion about the lowtemperature value of $r$. It is also known ${ }^{10}$ that ionized impurity scattering lowers the value of $r$, but at concentrations of $10^{13} \mathrm{~cm}^{-3},{ }^{10}$ this should be a rather small effect. Accepting Beer and Willardson's result, ${ }^{10} r=1.36$ \pm 0.07 at $81^{\circ} \mathrm{K}$, to represent pure acoustic scattering, an inspection of Fig. 5 shows that this can only be fitted by the theory if $a, B b$, and $D d$ have the same sign, which is at variance with the results of the more direct deter-

${ }^{33}$ O. A. Golikova and L. S. Stil'bans, Fiz. Tverd. Tela 3, 3115 (1961) [English transl.: Soviet Phys.--Solid State 3, 2266 (1962)]

${ }^{34}$ F. J. Morin, Phys. Rev. 93, 62 (1954); M, B, Prince, ibid. 92, 681 (1953).

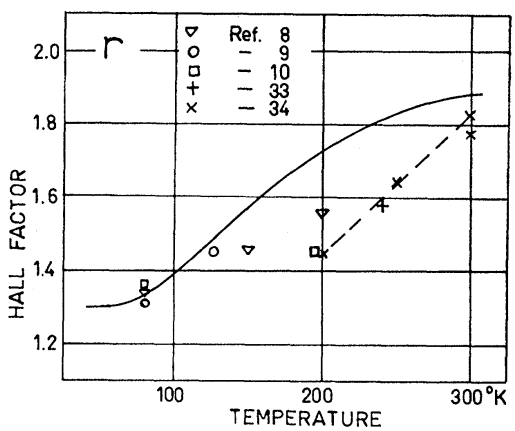

FIG. 14. Experimental data on the Hall factor $r$ selected to represent low magnetic field and pure lattice scattering. The full curve shows the minimum theoretical Hall factor also displayed in Fig. 10.

mination of $a, b$, and $d$ described above. The minimum value of $r_{\mathrm{ac}}$ for $|b| \approx 2 \mathrm{eV},|d| \approx 7 \mathrm{eV}$, and $a$ having opposite sign of $B b$ and $D d$ is about 1.60 , which is evidently far above any existing experimental value of $r_{\text {ac. }}$ The situation is not better at higher temperatures, as is indicated by a comparison between the experimental Fig. 14 and the theoretical Fig. 10. Thus, even if we suggest that $r_{\mathrm{ac}} \approx 1.6$ and explain the low-temperature discrepancy in terms of impurity scattering, this cannot be done at $300^{\circ} \mathrm{K}$, and we conclude that at least the theory of optical phonon scattering is incorrect.

Much the same arguments apply to the transverse magnetoresistance $b_{B}$ or $b_{B}{ }^{\prime}=b_{B} \mu_{0}^{-2}$. Here, relevant experimental data are scarce. At $81^{\circ} \mathrm{K}$, Beer and Willardson $^{12}$ give $b_{B}=6 \times 10^{9} \mathrm{~cm}^{4} / \mathrm{V}^{2} \mathrm{sec}^{2}$. Using the value of the mobility measured in Ref. $10, \mu_{81}=4.0 \times 10^{4} \mathrm{~cm}^{2} / \mathrm{V} \mathrm{sec}$, we find $b_{B}{ }^{\prime}=3.7$, which can only be fitted in the first quadrant of Fig. 6, just as is the case of the Hall factor. However, $b_{B}{ }^{\prime}=3.7$ may not actually represent pure lattice scattering, ${ }^{10}$ and the value suggested by the directly determined deformation potentials is $b_{B}{ }^{\prime} \approx 7.7$. The difference is seen to be quite large. At $196^{\circ} \mathrm{K}, \mathrm{Re}^{\mathrm{f}}$. 10 gives $b_{B}=2.5 \times 10^{8} \mathrm{~cm}^{4} / \mathrm{V}^{2} \mathrm{sec}^{2}$, and the corresponding reduced parameter is $b_{B}{ }^{\prime}=6.0\left(\mu_{196}=6.5 \times 10^{3} \mathrm{~cm}^{2} / \mathrm{V}\right.$ $\mathrm{sec})$. Finally, we add a value of $b_{B}=5 \times 10^{7} \mathrm{~cm}^{4} / \mathrm{V}^{2} \mathrm{sec}^{2}$ obtained by Glicksman ${ }^{35}$ at $290^{\circ} \mathrm{K}$ corresponding to $b_{B}{ }^{\prime}=8.2$. Neither of these values can be fitted in Fig. 11 if $b_{B}{ }^{\prime}=7.7$ is taken as the low-temperature value representing pure acoustic scattering. This gives a further indication that the theory of optical phonon scattering needs reconsideration.

Very little quantitative data exists concerning the longitudinal magnetoresistance parameters. From Goldberg et al., ${ }^{8}$ we derive $\left(\beta_{B}+\gamma_{B}+\frac{1}{2} \delta_{B}\right) / \beta_{B} \approx 0.17$ and $\left(\beta_{B}+\gamma_{B}+\delta_{B}\right) / \beta_{B} \approx 0.07$ at $77^{\circ} \mathrm{K}$. Using $b_{B}{ }^{\prime} \approx 3.7$ and $r \approx 1.36$ (both derived from Ref. 10 as discussed above), we find $\beta_{B}{ }^{\prime} \approx-5.6$, and this gives $\left(b_{B}{ }^{\prime}+c_{B}{ }^{\prime}+\frac{1}{2} d_{B}{ }^{\prime}\right)$ $\approx 0.95$ and $\left(b_{B}{ }^{\prime}+c_{B}{ }^{\prime}+d_{B}{ }^{\prime}\right) \approx 0.39$. Since a simplified theory of impurity scattering cannot be applied to these

${ }^{35}$ M. Glicksman, in Progress in Semiconductors, edited by A. F. Gibson (Heywood and Co., Ltd., London, 1958), Vol. 3, p. 1. 
parameters [it is not only the momentum relaxation time $\left(\Gamma_{15}\right)$ that enters the calculation], the influence of this type of scattering cannot be estimated in the present case, and no reliable values pertaining to pure acoustic scattering can be deduced from the above data. At $290^{\circ} \mathrm{K}$, the results of Glicksman ${ }^{35}$ indicate that $\left(b_{B}{ }^{\prime}+c_{B}{ }^{\prime}+\frac{1}{2} d_{B}{ }^{\prime}\right) \approx 1.2$, which is not inconsistent with Fig. 14 for a low-temperature value of 0.95 . The same applies to the rather uncertain value of $\left(b_{B}{ }^{\prime}+c_{B}{ }^{\prime}+d_{B}{ }^{\prime}\right)$ derived from Ref. 35 for $290^{\circ} \mathrm{K}$.

If we allow for ionized impurity scattering to explain the low values of $r$ and $b_{B}{ }^{\prime}$ at $77^{\circ} \mathrm{K}$, we can conclude from the above discussion that the acoustic scattering may be described by a deformation-potential theory using $|b| \approx 2 \mathrm{eV},|d| \approx 7 \mathrm{eV}$, and $a=(-1.5 \pm 1.5) \mathrm{eV}$ in agreement with results of experiments on strained $p$-Ge. To substantiate this claim further, there is a clear need for a theory of ionized impurity scattering of holes since the difference between measured and calculated values of $r$ and $b_{B}{ }^{\prime}$ is unexpectedly large for impurity concentrations of $10^{13} \mathrm{~cm}^{-3} \cdot{ }^{10} \mathrm{~A}$ first step in this direction is the theory of the transition rates given in Ref. 5. Further work on the subject is presently in progress. On the other hand, it is evident that optical phonon scattering is not well described by the usual theory.

\section{CONCLUSION}

In this paper, we have attempted to calculate some low-field transport properties of holes in Ge for pure lattice scattering, taking into account all relevant details of the band structure and of the scattering. It has thus been established that the nonparabolicity of the light hole band has a negligible effect below $300^{\circ} \mathrm{K}$, except when very high accuracy is wanted. Even in this case the effect may be overshadowed by secondary effects not considered in the present work.

The phonon scattering is described by the four deformation potentials $a, b, d$, and $d_{0}$. With the exception of mobility, existing experimental data on galvanomagnetic properties cannot be reconciled with directly determined deformation potentials without assuming an unexpectedly large influence of ionized impurity scat- tering. At room temperature, this is quite unlikely to be the case and we conclude that at least the theory of optical phonon scattering must be reconsidered. As evident from Table I, the values of $d$ determined directly from experiments on strained $\mathrm{Ge}$ show a significant scatter between different experimental methods. This suggests that also the acoustic or static part of the usual general form of the deformation-potential theory may have to be modified.

A theory giving nontrivial corrections to the deformation-potential approach to acoustic and optical phonon scattering has been put forward by Tolpygo. ${ }^{36}$ The basic idea is that long-range potentials of essentially quadrupole nature ${ }^{37}$ are induced by a vibration of the nonpolar crystal, and that these potentials are of the same order of magnitude as the deformation potential, but of a different angular dependence. Tolpygo's theory ${ }^{36}$ is based on a particular model of the lattice vibrations, but in a forthcoming paper we shall present a general theory of this long-range interaction and its relation to the ordinary deformation-potential model. This theory yields qualitatively the same results as Tolpygo obtained for the scattering but, in addition, it can explain the variation of $d$ values obtained by different static strain experiments. It may finally be added that the new theory gives a dramatic correction to optical phonon scattering which will no longer be momentum-randomizing.

Because of an error in the calculation of the factor in Eq. (4.7), all fitted values of the optical deformation potential $d_{0}$ should be multiplied by $2 \pi$.

\section{ACKNOWLEDGMENTS}

The author is indebted to Professor N. I. Meyer for suggesting this subject and for his continued encouragement during all stages of this work. Computerfacilities were kindly provided by the Northern Europe University Computing Center (NEUCC).

${ }^{36}$ K. B. Tolpygo, Fiz. Tverd. Tela 4, 1765 (1962) [English transl.: Soviet Phys.-Solid State 4, 1297 (1963)].

${ }^{37} \mathrm{M}$. Lax, in Proceedings of the International Conference on Lattice Dynamics, Copenhagen, 1963 (Pergamon Press, Inc., New York, 1965), p. 179. 